\title{
GROWTH RATES FOR THE LINEARIZED MOTION OF 3-D FLUID INTERFACES WITH SURFACE TENSION FAR FROM EQUILIBRIUM*
}

\author{
THOMAS Y. HOU ${ }^{\dagger}$ AND PINGWEN ZHANG ${ }^{\ddagger}$
}

\begin{abstract}
We consider the motion of two-fluid interfacial flows with surface tension in three space dimensions. We assume that the flow is inviscid, irrotational, and incompressible. In this case, one can reduce the entire motion to a boundary integral formulation involving variables defined on the free surface alone. We analyze the growth rates for the linearized motion about an arbitrary smooth solution, even far from equilibrium. Our analysis shows that surface tension provides a dispersive regularization for high frequency modes. But there are finite number of low frequency modes that can still grow exponentially in time due to the Kelvin-Helmholtz and Rayleigh-Taylor instabilities. Without surface tension regularization, the problem is linearly ill-posed, even for the stably stratified flow. With finite surface tension, we prove that these equations are well-posed in the appropriate Sobolev spaces. This result has a direct relevance to the stability analysis of the corresponding boundary integral method.
\end{abstract}

1. Introduction. We are concerned with the motion of an interface separating two fluids with surface tension in three dimensions. We assume that the flow is inviscid, irrotational and incompressible, so that the entire motion is determined by variables on the surface. In general, without additional regularizing effects such as surface tension or viscosity, the flow can be subject to the Rayleigh-Taylor or the Kelvin-Helmholtz instabilities. This will lead to unbounded growth in high frequency wave numbers. In this case, the problem is not well-posed in the Hadamard sense. Surface tension is known to be a dispersive regularization of the Rayleigh-Taylor and the Kelvin-Helmholtz instabilities near equilibrium (Drazin \& Reid [11]). However, in the case of two-fluid interfaces, the interfaces will typically deform significantly before strong nonlinear effects such as singularity formation become important. Therefore, it is not clear that if the regularization effect of surface tension obtained near the equilibrium still applies when the interface is deformed significantly from the equilibrium.

In this paper, we analyze the growth rates for the linearized motion of 3-D interfaces with surface tension about an arbitrary smooth solution, even far from equilibrium. Our analysis shows that surface tension indeed provides a dispersive regularization for high frequency modes even when the interface is significantly deformed. But there are finite number of low frequency modes that can still grow exponentially in time due to the Kelvin-Helmholtz and Rayleigh-Taylor instabilities. Through nonlinear interactions, the number of unstable modes can increase rapidly in time. This may lead to singularity formation in finite time. Indeed, new topological singularities have been observed numerically for 2-D interfaces with surface tension $[14,15,10]$. It is worth mentioning that without surface tension regularization, the problem is linearly ill-posed, even for the stably stratified flow ( see, e.g. [5] and Corollary 4.1 of this paper). With finite surface tension, we prove that the two-fluid interface problem is well-posed in the appropriate Sobolev spaces. This result has a direct relevance to the stability analysis of the corresponding boundary integral method.

\footnotetext{
${ }^{*}$ Received November 6, 1997; accepted for publication June 27, 1998.

† Department of Applied Mathematics, California Institute of Technology, Pasadena, CA 91125, USA (hou@ama.caltech.edu).

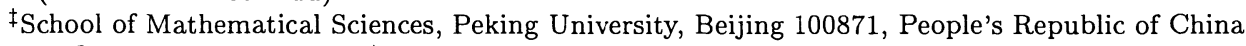
(pzhang@sxx0.math.pku.edu.cn).
} 
The analysis is based on the general framework developed in [3] and [16]. As in our previous studies, it is essential to project the linearized system to the tangent and normal vectors of the unperturbed smooth interface. The linearized system admits a beautiful structure when viewed in these coordinates. There are several new difficulties that we need to overcome here. The first one is that surface tension only provides regularization for high frequency components. Low frequency modes are still subject to the Kelvin-Helmholtz and the Rayleigh-Taylor instabilities. In order to see clearly how surface tension stabilizes the Kelvin-Helmholtz instability, we perform a change of variables to transfer the destabilizing terms to the same equation that contains the surface tension term. This makes it very clear how to perform energy estimates to control the high frequency modes and how to compensate for the potential growth from the low frequency modes. The second difficulty is due to the presence of two tangential dimensions. Only one of these tangential components is responsible for introducing fluid dynamical instability to the low modes. The other one only corresponds to the translation invariance along the tangent plane. We introduce additional change of variables to separate these two components, resulting in a system qualitatively similar to that of the corresponding 2-D case $[5,6]$. The last and most important difficulty is due to the three dimensionality. Since the 3-D singular kernel has a branch point singularity, we no longer have infinite order smoothing operators for the lower order terms. Instead, we only have an order one smoothing operator in $\gamma$ in the linearized equation. This introduces an essential difficulty in closing the energy estimates. We overcome this difficulty by performing energy estimates using the dipole strength variable, $\dot{\mu}$, instead of using the vortex sheet strength variables, $\gamma_{i}(i=1,2)$. By doing this, we reduce the total number of equations by one. Moreover, we incorporate the order one smoothing operator in $\gamma$ into our energy functional. The final energy estimates can be obtained by using the $H^{1}$ norm estimate for the interface variable, $\mathbf{z}$, and the $H^{1 / 2}$ norm for the dipole strength variable, $\mu$.

Our present study is largely motivated by the related stability analysis of boundary integral methods for computing interfacial flows (see e.g. $[1,2,3,9,10,12,14$, $15,17,21,22]$ and the recent review paper [13]). One of the purposes is to provide an analytical framework to analyze numerical stability of boundary integral methods in three space dimensions. Our previous study for the 2-D water wave problem indicates that boundary integral methods must satisfy certain compatibility condition in order to be stable [4]. At the continuous level, various singular operators are related to one another by certain compatibility conditions. It turns out that a similar compatibility must be enforced at the discrete level in order to obtain stability. Violation of this compatibility condition will lead to numerical instability. This has been one of the main difficulties in using boundary integral methods for computing free boundary problems.

The organization of the paper is as follows. In Section 2 we derive the boundary integral formulation for the two-fluid interface in three dimensions. The main results are stated in Section 3. In Section 4, we derive the linearized equations for the twofluid interface equations. Finally we prove the well-posedness of the linearized motion by careful energy estimates in Section 5 .

2. Derivation of the boundary integral formulation. In this section, we give a brief review of the derivation of the boundary integral formulation for 3-D interfacial flows. Throughout the paper, we will use bold face letter to denote vector variables. We assume that the flow is inviscid, incompressible, irrotational, and is separated by a free interface. In each fluid, the flow is governed by the incompressible 
Euler equations:

$$
\frac{\partial \mathbf{u}_{i}}{\partial t}+\left(\mathbf{u}_{i} \cdot \nabla\right) \mathbf{u}_{i}=-\frac{1}{\rho_{i}} \nabla p_{i}-\mathbf{g}, \quad i=1,2 .
$$

The incompressibility constraint is

$$
\nabla \cdot \mathbf{u}_{i}=0, \quad i=1,2 .
$$

Irrotationality gives

$$
\nabla \times \mathbf{u}_{i}=0, \quad i=1,2 .
$$

We impose Laplace-Young's boundary condition across the interface which relates the pressure jump to the mean curvature of the interface, $\kappa$, by

$$
p_{1}-p_{2}=-\tau \kappa
$$

where $p_{1}, p_{2}$ are the limiting pressures from below and above the interface respectively, $\tau$ is the surface tension coefficient. We also require the interface to move with the fluid. This gives the additional boundary condition

$$
\left(\mathbf{u}_{1}-\mathbf{u}_{2}\right) \cdot \mathbf{N}=0
$$

where $\mathbf{N}$ is the unit upward normal vector to the interface, $\mathbf{u}_{1}, \mathbf{u}_{2}$ are the limiting velocities from below and above the interface respectively. Since the flow is irrotational, there exist velocity potentials $\phi_{i}$ such that

$$
\mathbf{u}_{i}=\nabla \phi_{i}, \quad i=1,2 .
$$

Incompressibility then gives

$$
\operatorname{div} \cdot \mathbf{u}_{i}=\triangle \phi_{i}=0, \quad i=1,2 .
$$

To update the velocity potential in time, we use Bernoulli's equation:

$$
\frac{\partial \phi_{i}}{\partial t}+\frac{\mathbf{u}_{i} \cdot \mathbf{u}_{i}}{2}+\frac{p_{i}}{\rho_{i}}+\mathbf{g} \cdot \mathbf{z}=0, \quad i=1,2
$$

where $\mathbf{g}$ is the gravity acceleration vector, and $\mathbf{z}$ is the interface position. Note that the tangential velocity may be discontinous. It is convenient to use the average of the velocities above and below the interface as the interface velocity. Denote by $\mathbf{U}$ the interface velocity defined on the interface $\Gamma$. Then it is given by

$$
\mathbf{U}=\left(\mathbf{u}_{1}+\mathbf{u}_{2}\right) / 2=\left\{\left(\nabla \phi_{1}\right)+\left(\nabla \phi_{2}\right)\right\} / 2 .
$$

We parametrize the interface $\Gamma$ at time $t$ by $\mathbf{z}=\mathbf{z}(\alpha, t)$, where $\alpha=\left(\alpha_{1}, \alpha_{2}\right)$. Then $\mathbf{z}$ satisfies

$$
\begin{aligned}
\frac{\partial}{\partial t} \mathbf{z}(\alpha, t) & =\mathbf{U}(\mathbf{z}(\alpha, t)), \\
\mathbf{z}(\alpha, 0) & =\mathbf{z}_{0}(\alpha) .
\end{aligned}
$$

Let the dipole strength $\mu(\alpha, t)$ be defined by

$$
\mu(\alpha, t)=\phi_{1}(\mathbf{z}(\alpha, t), t)-\phi_{2}(\mathbf{z}(\alpha, t), t) .
$$


Then $\mu$ satisfies

$$
\frac{\partial \mu}{\partial t}=\left[\frac{\partial \phi}{\partial t}\right]+\mathbf{U} \cdot[\nabla \phi]
$$

On the other hand, by Bernoulli's equation (8), we have

$$
\left[\frac{\partial \phi}{\partial t}\right]+\frac{[\mathbf{u} \cdot \mathbf{u}]}{2}+\left(\frac{p_{1}}{\rho_{1}}-\frac{p_{2}}{\rho_{2}}\right)=0
$$

Note that

$$
\frac{[\mathbf{u} \cdot \mathbf{u}]}{2}=\frac{\left(\mathbf{u}_{1}+\mathbf{u}_{2}\right)}{2} \cdot\left(\mathbf{u}_{1}-\mathbf{u}_{2}\right)=\mathbf{U} \cdot[\nabla \phi]
$$

It follows from (13) and (14) that,

$$
\frac{\partial \mu}{\partial t}=\frac{p_{2}}{\rho_{2}}-\frac{p_{1}}{\rho_{1}}=\frac{\tau \kappa}{\rho_{1}}+\frac{\rho_{1}-\rho_{2}}{\rho_{1} \rho_{2}} p_{2} .
$$

By differentiating (15) with respect to $\alpha_{i}$, we get

$$
\frac{\partial \mu_{\alpha_{i}}}{\partial t}=\frac{\tau}{\rho_{1}} \kappa_{\alpha_{i}}+\frac{\rho_{1}-\rho_{2}}{\rho_{1} \rho_{2}}\left(p_{2}\right)_{\alpha_{i}}, \quad i=1,2 .
$$

We now can write the potential in the fluid domain in terms of $\mu[1,2]$

$$
\begin{aligned}
\phi(\mathbf{z}) & =\int \mu\left(\alpha^{\prime}\right)\left(\mathbf{z}_{\alpha_{1}} \times \mathbf{z}_{\alpha_{2}}\right)\left(\alpha^{\prime}\right) \nabla_{\mathbf{z}^{\prime}} G\left(\mathbf{z}-\mathbf{z}\left(\alpha^{\prime}\right)\right) d \alpha^{\prime} \\
& \equiv K \mu
\end{aligned}
$$

where

$$
G\left(\mathbf{z}-\mathbf{z}^{\prime}\right)=-\frac{1}{4 \pi\left|\mathbf{z}-\mathbf{z}^{\prime}\right|}, \quad \nabla_{\mathbf{z}^{\prime}} G\left(\mathbf{z}-\mathbf{z}^{\prime}\right)=-\frac{\mathbf{z}-\mathbf{z}^{\prime}}{4 \pi\left|\mathbf{z}-\mathbf{z}^{\prime}\right|^{3}} .
$$

Differentiating both sides of the $\phi(\mathbf{z})$ equation with respect to $\mathbf{z}$ and integrating by parts, we obtain

$$
\mathbf{u}=\nabla \phi(\mathbf{z})=\int \mathbf{W} \times \nabla_{\mathbf{z}^{\prime}} G\left(\mathbf{z}-\mathbf{z}\left(\alpha^{\prime}\right)\right) d \alpha^{\prime}
$$

where $\mathbf{W}=J \boldsymbol{\Omega}=\mu_{\alpha_{1}} \mathbf{z}_{\alpha_{2}}-\mu_{\alpha_{2}} \mathbf{z}_{\alpha_{1}}$, and $\boldsymbol{\Omega}=\mathbf{N} \times[\nabla \phi], J=\left|\mathbf{z}_{\alpha_{1}} \times \mathbf{z}_{\alpha_{2}}\right|$. Letting $\mathbf{z}$ approach to the interface from above and from below the interface, one obtains [12]

$$
\begin{aligned}
& \mathbf{u}_{1}=\mathbf{U}+\frac{1}{2} \frac{\mathbf{W} \times \mathbf{N}}{J} \\
& \mathbf{u}_{2}=\mathbf{U}-\frac{1}{2} \frac{\mathbf{W} \times \mathbf{N}}{J}
\end{aligned}
$$

and the average velocity $\mathbf{U}$ is given by

$$
\mathbf{U}(\alpha, t)=\int \mathbf{W} \times \nabla_{\mathbf{z}^{\prime}} G\left(\mathbf{z}(\alpha)-\mathbf{z}\left(\alpha^{\prime}\right)\right) d \alpha^{\prime} .
$$


Taking into account the Lagrangian variables and using Bernoulli's theorem (8), we have

$$
\frac{\partial\left(\phi_{2}+\phi_{1}\right)}{\partial t}-2|\mathbf{U}|^{2}+\frac{1}{2}\left(\left|\mathbf{u}_{1}\right|^{2}+\left|\mathbf{u}_{2}\right|^{2}\right)-\frac{\tau \kappa}{\rho_{1}}+\frac{\rho_{1}+\rho_{2}}{\rho_{1} \rho_{2}} p_{2}+2 \mathbf{g} \cdot \mathbf{z}=0 .
$$

Combining (15) and (21), we have

$$
\frac{\partial \mu}{\partial t}=-A\left(\frac{\partial\left(\phi_{2}+\phi_{1}\right)}{\partial t}-2|\mathbf{U}|^{2}+\frac{1}{2}\left(\left|\mathbf{u}_{1}\right|^{2}+\left|\mathbf{u}_{2}\right|^{2}\right)+2 \mathbf{g} \cdot \mathbf{z}\right)+\frac{\tau}{\rho_{1}}(1+A) \kappa
$$

where $A=\left(\rho_{1}-\rho_{2}\right) /\left(\rho_{1}+\rho_{2}\right)$ is the Atwood number. By differentiating (22) with respect to $\alpha_{i}$, we obtain

$$
\begin{aligned}
& \frac{\partial \mu_{\alpha_{i}}}{\partial t}=-A\left(2 \frac{\partial}{\partial t}\left(\mathbf{U} \cdot \mathbf{z}_{\alpha_{i}}\right)-2|\mathbf{U}|_{\alpha_{i}}^{2}+\left(\mathbf{u}_{1} \cdot\left(\mathbf{u}_{1}\right)_{\alpha_{i}}+\mathbf{u}_{2} \cdot\left(\mathbf{u}_{2}\right)_{\alpha_{i}}\right)+2 \mathbf{g} \cdot \mathbf{z}_{\alpha_{i}}\right) \\
& +\frac{\tau}{\rho_{1}}(1+A) \kappa_{\alpha_{i}} . \quad i=1,2
\end{aligned}
$$

Using (10), (18) and (19), we have

(24) $\frac{\partial \gamma_{i}}{\partial t}=-2 A\left(\frac{\partial^{2} \mathbf{z}}{\partial t^{2}} \cdot \mathbf{z}_{\alpha_{i}}+\frac{1}{8}\left|\frac{\mathbf{W} \times \mathbf{N}}{J}\right|_{\alpha_{i}}^{2}+\mathbf{g} \cdot \mathbf{z}_{\alpha_{i}}\right)+\frac{\tau}{\rho_{1}}(1+A) \kappa_{\alpha_{i}}, \quad i=1,2$,

where $\gamma_{i}=\mu_{\alpha_{i}}$. Hereafter, we will denote $\frac{\tau}{\rho_{1}}(1+A)$ by $\tau$, where $-1<A<1$. In the special case of $A=-1$, only the Rayleigh-Taylor instability exists. The well-posedness of the linearized motion has been obtained by Zhang [27].

3. Statement of main results. We now state our well-posedness result for the linearized motion of equations (10), (20) and (24) far from equilibrium. We will need the following assumptions on the underlying smooth solution.

Necessary Properties of Smooth Solution: For $0 \leq t \leq T$,

(1) the interface can not self-intersect, i.e. $\mathbf{z}(\alpha, t) \neq \mathbf{z}\left(\alpha^{\prime}, t\right)$ for $\alpha \neq \alpha^{\prime}$;

(2) $\mathbf{z}(\alpha, t)-\left(\alpha_{1}, \alpha_{2}, 0\right)$ is continuous in time with values in $H^{m}$ with $m \geq 4$;

(3) $\mathbf{z}_{\alpha_{i}}(\alpha, t) \neq 0, i=1,2$ for all $\alpha$.

These are rather mild assumptions about the underlying solutions and they have the following important consequences. Properties (2) and (3) imply the existence of $c_{0}$ and $C_{0}$ such that

$$
\left|\mathbf{z}_{\alpha_{i}}(\alpha, t)\right| \geq c_{0}>0, \quad \text { as } \quad\left|\mathbf{z}(\alpha, \mathrm{t})-\mathbf{z}\left(\alpha^{\prime}, \mathrm{t}\right)\right| \geq \mathrm{C}_{0}\left|\alpha-\alpha^{\prime}\right|,
$$

for all $\alpha, \alpha^{\prime}, t$. We are now ready to present our main result.

Theorem 3.1. (Linear Well-Posedness Far from Equilibrium) Let $\mathbf{z}$ and $\gamma$ be the smooth solution of Eqs. (10), (20) and (24) satisfying the properties (1)-(3) listed above. Then for $0 \leq t \leq T$, there exists a constant $M$ such that the solution $\dot{\mathbf{z}}, \dot{\gamma}$ of the linearized equations (35)-(38) around $\mathbf{z}$ and $\gamma$ satisfies

$$
\|\dot{\mathbf{z}}(\cdot, t)\|_{H^{1}}+\|\dot{\mu}(\cdot, t)\|_{H^{1 / 2}} \leq M\left(\|\dot{\mathbf{z}}(\cdot, 0)\|_{H^{1}}+\|\dot{\mu}(\cdot, 0)\|_{H^{1 / 2}}\right)
$$

It is instructive to consider the linear stability of equilibrium solutions for Eqs. (10), (20) and (24). From the classical stability analysis, it is known that surface 
tension is a dispersive regularization near equilibrium. This is demonstrated below. The equilibrium solution is the flat interface with constant vortex sheet strength:

$$
\mathbf{z}^{e}(\alpha, t)=\left(\alpha_{1}, \alpha_{2}, 0\right) \quad \text { and } \quad \gamma_{\mathrm{i}}^{\mathrm{e}}(\alpha, \mathrm{t})=\gamma_{\mathrm{i}}^{0}, \quad \mathrm{i}=1,2,
$$

where $\gamma_{i}^{0}$ are constants. We look for solutions of (10), (20) and (24) of the form $\mathbf{z}=\mathbf{z}^{e}+\varepsilon \dot{\mathbf{z}}$ and $\gamma_{i}=\gamma_{i}^{e}+\varepsilon \dot{\gamma}_{i}$. Keeping only the $O(\varepsilon)$ terms gives the linearized equations

$$
\begin{aligned}
\frac{\partial \dot{x}}{\partial t}= & -\frac{1}{2}\left(\gamma_{1}^{0} H_{1} D_{1}(\dot{z})+\gamma_{2}^{0} H_{2} D_{1}\right)(\dot{z}) \\
\frac{\partial \dot{y}}{\partial t}= & -\frac{1}{2}\left(\gamma_{1}^{0} H_{1} D_{2}(\dot{z})+\gamma_{2}^{0} H_{2} D_{2}\right)(\dot{z}) \\
\frac{\partial \dot{z}}{\partial t}= & \frac{1}{2}\left(H_{1}\left(\dot{\gamma}_{1}\right)+H_{2}\left(\dot{\gamma}_{2}\right)\right) \\
& -\frac{1}{2}\left(\gamma_{1}^{0}\left(H_{1} D_{1}+H_{2} D_{2}\right)(\dot{x})+\gamma_{2}^{0}\left(H_{1} D_{1}+H_{2} D_{2}\right)(\dot{y})\right) \\
\frac{\partial \dot{\gamma}_{i}}{\partial t}= & A\left\{\left(\left[\left(\gamma_{1}^{0}\right)^{2} D_{1}+\gamma_{1}^{0} \gamma_{2}^{0} D_{2}\right] D_{i}(\dot{x})+\left[\gamma_{1}^{0} \gamma_{2}^{0} D_{1}+\left(\gamma_{2}^{0}\right)^{2} D_{2}\right] D_{i}(\dot{y})\right.\right. \\
& -2 g \dot{z}_{\alpha_{i}}+\frac{1}{2}\left[\gamma_{1}^{0} H_{1}^{2}+\gamma_{2}^{0} H_{1} H_{2}-\gamma_{1}^{0}\right] D_{i}\left(\dot{\gamma}_{1}\right) \\
& \left.\left.+\frac{1}{2}\left[\gamma_{2}^{0} H_{2}^{2}+\gamma_{1}^{0} H_{1} H_{2}-\gamma_{2}^{0}\right] D_{i}\left(\dot{\gamma}_{2}\right)\right\}\right) \\
& +\frac{\tau}{2} D_{i}\left(\dot{z}_{\alpha_{1} \alpha_{1}}+\dot{z}_{\alpha_{2} \alpha_{2}}\right) . \quad i=1,2 .
\end{aligned}
$$

The operator $H_{i}$ is the Hilbert transform defined on the flat plane

$$
H_{i}(f)=\frac{1}{2 \pi} \int \frac{\left(\alpha_{i}-\alpha_{i}^{\prime}\right) f\left(\alpha^{\prime}\right)}{\left|\alpha-\alpha^{\prime}\right|^{3}} d \alpha^{\prime} . \quad i=1,2 .
$$

The system is easily diagonalized in Fourier transform space. Let

$$
\hat{f}(k)=\int f(\alpha) e^{-i k \cdot \alpha} d \alpha .
$$

The inverse transform reads

$$
f(\alpha)=\frac{1}{(2 \pi)^{2}} \int \hat{f}(k) e^{i k \cdot \alpha} d \alpha .
$$

Then it is easy to show [16] that $\hat{H}_{l}=-i \frac{k_{l}}{|k|}, l=1,2$. The eigenvalues of the transformed system are

$$
\omega(k)=0,0,0, \frac{i}{2} A \widetilde{k} \pm \frac{1}{2} \sqrt{\tilde{k}^{2}\left(1-A^{2}\right)-2 A g|k|-\tau|k|^{3}},
$$

where $i^{2}=-1$, and $\widetilde{k}=\gamma_{1}^{0} k_{1}+\gamma_{2}^{0} k_{2}$. The $\omega=0$ growth rate simply reflects that translating Lagrangian points along the interface does not change its shape. If the surface tension coefficient $\tau$ is small, there will be finite number of unstable modes with positive real part. The amplitude of these unstable modes will grow exponentially in time. However, for high frequency modes with large $|k|$, the eigenvalues become purely imaginary. Imaginary growth rate produces oscillations rather than growth. 
Therefore, while the lower modes can grow, the high modes just oscillate. This results in an overall bounded growth (in $k$ ) of the solution. On the other hand, if $\tau=0$ then the growth can be exponential in the wave number $k$ due to the Kelvin-Helmholtz and Rayleigh-Taylor instabilities. Surface tension acts as a dispersive regularization of these frequency instabilities.

4. Derivation of linearized equations far from equilibrium. As we know from the equilibrium stability analysis, surface tension gives a dispersive regularization to the Rayleigh-Taylor and the Kelvin-Helmholtz instabilities near equilibrium. However, in the case of two-fluid interfaces, the interfaces typically deform significantly from the equilibrium before strong nonlinear effects such as singularity formation become important. Therefore, it is natural to ask if the regularization effect of surface tension obtained near the equilibrium still applies when the interface is deformed significantly from the equilibrium. To answer this question, we study the growth rates of the linearized motion around an arbitrary smooth solution far from equilibrium.

Let $\mathbf{z}(\alpha, t)$ and $\gamma_{i}(\alpha, t)$ be the smooth, time dependent solution to (10), (20) and (24). Denote by $\dot{\mathbf{z}}$ and $\dot{\gamma}_{i}$ the perturbations in $\mathbf{z}$ and $\gamma_{i}$ respectively. Replace $\mathbf{z}, \gamma_{i}$ in (10), (20) and (24) by $\mathbf{z}+\varepsilon \dot{\mathbf{z}}, \gamma_{i}+\varepsilon \dot{\gamma}_{i}$. To prove the well-posedness of the linearized motion around an arbitrary smooth interface, we would like to demonstrate that the perturbed quantities in some appropriate Sobolev norm at later times can be bounded by the initial perturbations in the same Sobolev norm. The linearization and the well-posedness analysis will be carried out in the Lagrangian frame. Since the linearized equations contain the Rietz transform and its variants defined on a free surface, the analysis becomes quite complicated. To simplify the presentation and to emphasize the key points in the analysis, we will make a change of variables in the parametrization of the unperturbed solution from $\alpha$ to $\beta$, after we obtain the linearized equations. It is worth emphasizing that this change of variables in the parametrization is done after the linearized equations have been obtained. If we make this change of variables before the linearization, this will amount to performing linearization on a different set of equations, which is in general not consistent with the Lagrangian frame. We remark that the analysis can also be performed in the original Lagrangian frame without making this change of variables in the parametrization. The analysis can proceed following the general framework laid out in this paper. The analysis would be more complicated because the properties of the corresponding singular operators are not as elegant as the ones using the orthogonal parametrization.

We choose $\beta_{1}$ and $\beta_{2}$ to be the orthogonal coordinates [16] on the surface so that

$$
\mathbf{z}_{\beta_{1}} \cdot \mathbf{z}_{\beta_{2}}=0 .
$$

Define the tangent and normal vectors of the smooth interface by

$$
\mathbf{T}_{i}=\sigma_{i} \mathbf{z}_{\beta_{i}} \quad i=1,2, \quad \text { and } \quad \mathbf{N}=\mathbf{T}_{1} \times \mathbf{T}_{2},
$$

where $\sigma_{i}=\left|z_{\beta_{i}}\right|^{-1}, i=1,2$. We denote the change of variable from $\alpha$ to $\beta$ as $\alpha=\alpha(\beta, t)$, which is in general time dependent. Let $Q$ be the Jacobian matrix $\frac{\partial\left(\alpha_{1}, \alpha_{2}\right)}{\partial\left(\beta_{1}, \beta_{2}\right)}$. Define $\tilde{\mathbf{z}}(\beta, t)=\mathbf{z}(\alpha(\beta, t), t)$ and $\tilde{\gamma}_{i}(\beta, t)=\gamma_{i}(\alpha(\beta, t), t)$. Then the partial derivative in time is given by

$$
\begin{aligned}
\frac{\partial \tilde{\mathbf{z}}(\beta, t)}{\partial t} & =\frac{\partial \mathbf{z}(\alpha, t)}{\partial t}+\frac{\partial \alpha(\beta, t)}{\partial t} \cdot \nabla_{\alpha} \mathbf{z} \\
& =\frac{\partial \mathbf{z}(\alpha, t)}{\partial t}+\left(\frac{\partial \alpha(\beta, t)}{\partial t} Q^{-1}\right) \cdot \nabla_{\beta} \tilde{\mathbf{z}}
\end{aligned}
$$


We introduce a new time derivative operator, $D / D t$, to absorb the convection term induced by this change of variables:

$$
\frac{D \tilde{z}(\beta, t)}{D t}=\frac{\partial \tilde{z}(\beta, t)}{\partial t}-\left(\frac{\partial \alpha(\beta, t)}{\partial t} Q^{-1}\right) \cdot \nabla_{\beta} \tilde{z}
$$

In the rest of paper, after the linearized equations are obtained, we will parametrize the unperturbed surface by this orthogonal coordinate, $\beta$. The free surface variables $\tilde{z}$ and $\tilde{\mu}$ are now considered as a function of $\beta$. As in [16], this change of variables does not affect the integral representation of the interface velocity as well as the local velocity. We can simply switch from $\alpha$ to $\beta$ in the integrals. The leading order singular integral operators are greatly simplified. This makes it easier to perform energy estimates. To simplify the notations and the presentation, we will drop the tilde and still use $\alpha$ for $\beta$. Thus, $z_{\alpha_{1}}$ and $z_{\alpha_{2}}$ are now orthogonal tangent vectors (more precisely we are referring to the orthogonality of $\tilde{z}_{\beta_{1}}$ and $\tilde{z}_{\beta_{2}}$ ). Moreover, we would like to point out that since the Jacobian $\operatorname{det}(Q)$ is bounded and has bounded inverse, the $H^{s}(s \geq 0)$ norm of $\tilde{\dot{z}}(\beta)$ is equivalent to the $H^{s}$ norm of $\dot{z}(\alpha)$.

Notations. Throughout the paper, we will use $E_{l}$ to denote a bounded operator from $H^{s}$ to $H^{s+l}$. If $l \geq 0$, we call $E_{l}$ a linear operator of order $l$. If $l<0$, then we call $E_{l}$ a smoothing operator of order $|l|$. We also project $\dot{\mathbf{z}}$ into the tangent and normal coordinates as follows:

$$
\dot{z}^{T_{1}}=\dot{\mathbf{z}} \cdot \mathbf{T}_{1}, \quad \dot{z}^{T_{2}}=\dot{\mathbf{z}} \cdot \mathbf{T}_{2}, \quad \dot{z}^{N}=\dot{\mathbf{z}} \cdot \mathbf{N} .
$$

The linearized equations for $\dot{z}^{T_{1}}, \dot{z}^{T_{2}}, \dot{z}^{N}$ and $\dot{\gamma}_{i}$ are given by the following lemma:

LEMMA 4.1. (Linearized Equations Far from Equilibrium) Suppose that $\left|\mathbf{z}_{\alpha_{i}}\right| \geq c_{0}>0, i=1,2$. Then to the leading order, $O(\varepsilon)$, the linearized equations for $\dot{z}^{T_{1}}, \dot{z}^{T_{2}}, \dot{z}^{N}$ and $\dot{\gamma}_{1}, \dot{\gamma}_{2}$ are given as follows

$$
\begin{aligned}
\text { (35) } \frac{D \dot{z}^{T_{1}}}{D t}= & -\frac{1}{2}\left(\sigma_{2}\right)^{-1}\left(\gamma_{1} H_{1} D_{1}+\gamma_{2} H_{2} D_{1}\right)\left(\dot{z}^{N}\right)+K_{1}(\dot{\mu})+E_{0}(\dot{\mathbf{z}}) \\
\text { (36) } \frac{D \dot{z}^{T_{2}}}{D t}= & -\frac{1}{2}\left(\sigma_{1}\right)^{-1}\left(\gamma_{1} H_{1} D_{2}+\gamma_{2} H_{2} D_{2}\right)\left(\dot{z}^{N}\right)+K_{2}(\dot{\mu})+E_{0}(\dot{\mathbf{z}}) \\
\text { (37) } \frac{D \dot{z}^{N}}{D t}= & \frac{1}{2} \sigma_{1} \sigma_{2} \widetilde{\Lambda} \dot{\mu}-\frac{1}{2}\left(\sigma_{1} \gamma_{1} \widetilde{\Lambda}\left(\dot{z}^{T_{1}}\right)+\sigma_{2} \gamma_{2} \widetilde{\Lambda}\left(\dot{z}^{T_{2}}\right)\right)+E_{0}(\dot{\mathbf{z}}) \\
\text { (38) } \frac{D \dot{\gamma}_{l}}{D t}= & A\left\{\sigma_{1}\left[\gamma_{1}^{2} \sigma_{1}^{2} D_{1}+\gamma_{1} \gamma_{2} \sigma_{2}^{2} D_{2}\right] D_{l}\left(\dot{z}^{T_{1}}\right)+\sigma_{2}\left[\gamma_{1} \gamma_{2} \sigma_{1}^{2} D_{1}+\gamma_{2}^{2} \sigma_{2}^{2} D_{2}\right] D_{l}\left(\dot{z}^{T_{2}}\right)\right. \\
& -2 K_{l}\left(\frac{\partial \dot{\mu}}{\partial t}\right)+\frac{1}{2}\left[\left(\sigma_{1} \sigma_{2}\right)^{-2}\left(\gamma_{1} H_{1}^{2}+\gamma_{2} H_{1} H_{2}\right)-\gamma_{1} \sigma_{1}^{2}\right] D_{l}\left(\dot{\gamma}_{1}\right) \\
& \left.+\frac{1}{2}\left[\left(\sigma_{1} \sigma_{2}\right)^{-2}\left(\gamma_{2} H_{2}^{2}+\gamma_{1} H_{1} H_{2}\right)-\gamma_{2} \sigma_{2}^{2}\right] D_{l}\left(\dot{\gamma}_{2}\right)\right\} \\
& +\frac{\tau}{2} D_{l}\left\{\sigma_{1} D_{1}\left(\sigma_{1} D_{1} \dot{z}^{N}\right)+\sigma_{2} D_{2}\left(\sigma_{2} D_{2} \dot{z}^{N}\right)\right. \\
& \left.-\sigma_{1} \sigma_{2}\left(\Gamma_{1}^{12} \dot{z}_{\alpha_{2}}^{N}+\Gamma_{2}^{21} \dot{z}_{\alpha_{1}}^{N}\right)\right\} \\
& +E_{0}\left(D_{l} \dot{\mathbf{z}}\right)+E_{0}(\dot{\gamma}), \quad l=1,2,
\end{aligned}
$$

where $H_{l}$ are the Hilbert transforms defined on the interface,

$$
\left(H_{l} f\right)(\alpha)=\frac{1}{2 \pi} \int \frac{\left(\alpha_{l}-\alpha_{l}^{\prime}\right) f\left(\alpha^{\prime}\right)}{\left(\left|z_{\alpha_{1}}(\alpha)\right|^{2}\left(\alpha_{1}-\alpha_{1}^{\prime}\right)^{2}+\left|z_{\alpha_{2}}(\alpha)\right|^{2}\left(\alpha_{2}-\alpha_{2}^{\prime}\right)^{2}\right)^{3 / 2}} d \alpha^{\prime}
$$


for $l=1,2, K_{1}(\dot{\mu})=\mathbf{T}_{1} \cdot \mathbf{V}(\dot{\mu}), K_{2}(\dot{\mu})=\mathbf{T}_{2} \cdot \mathbf{V}(\dot{\mu})$, and $\tilde{\Lambda}=\left(\sigma_{1} \sigma_{2}\right)^{-1} \mathbf{N} \cdot \mathbf{V}(\dot{\mu})$, where $\mathbf{V}(\dot{\mu})$ is defined as

$$
\mathbf{V}(\dot{\mu})=\int\left(\dot{\mu}_{\alpha_{1}} \mathbf{z}_{\alpha_{2}}-\dot{\mu}_{\alpha_{2}} \mathbf{z}_{\alpha_{1}}\right) \times \nabla z^{\prime} G\left(\mathbf{z}(\alpha)-\mathbf{z}\left(\alpha^{\prime}\right)\right) d \alpha^{\prime}
$$

REMARK 4.1. Note that the $\widetilde{\Lambda}(\dot{\mu})$ term in the $\dot{z}^{N}$ equation contains all the contribution from $\dot{\mu}$. This eliminates the $E_{-1}(\dot{\gamma})$ from $\dot{z}^{N}$ equation (compare to the case of the water wave problem in [16]). This is the reason why we introduce a new $\tilde{\Lambda}$ operator in the $\dot{z}^{N}$ equation (see Lemma 4.3 in [16]). It is straightforward to show that

$$
\widetilde{\Lambda}(\dot{\mu})=\left(\sigma_{1} \sigma_{2}\right)^{-2}\left(H_{1} \dot{\mu}_{\alpha_{1}}+H_{2} \dot{\mu}_{\alpha_{2}}\right)+E_{0}(\dot{\mu})=\left(\sigma_{1} \sigma_{2}\right)^{-2} \Lambda \dot{\mu}+E_{0}(\dot{\mu}),
$$

where $\Lambda=H_{1} D_{1}+H_{2} D_{2}$ is given by

$$
(\Lambda f)(\alpha)=\int \frac{f(\alpha)-f\left(\alpha^{\prime}\right)}{\left(\left|z_{\alpha_{1}}(\alpha)\right|^{2}\left(\alpha_{1}-\alpha_{1}^{\prime}\right)^{2}+\left|z_{\alpha_{2}}(\alpha)\right|^{2}\left(\alpha_{2}-\alpha_{2}^{\prime}\right)^{2}\right)^{3 / 2}} d \alpha^{\prime} .
$$

By using integration by parts, we can show that $K_{i}(\dot{\mu})=D_{i} K(\dot{\mu}), i=1,2$.

Notice the remarkable similarity between the equations (35)-(38) and the linearized equations (28)-(31) near equilibrium. The equations (28)-(31) are essentially the frozen coefficient versions of (35)-(38). This is because the smooth interface is locally flat when viewed from its tangential and normal coordinates.

Further, notice that the gravity term is missing from the $\dot{\gamma}_{i}$ equation in (35)-(38). This is because the gravity term involves only one spatial derivative. It is included in the lower order term, $E_{0}\left(D_{l} \dot{\mathbf{z}}\right)$.

Similar to the two dimensional case, equations (35)-(38) have the following immediate consequence. It shows that if $\tau=0$, then the linearized equations for the perturbations are essentially ill-posed, even in the stably stratified case where the Atwood number $A>0$.

COROllary 4.1. Ill-Posedness of Linearized Equations for $\tau=0$. Let the smooth solution, $\mathbf{z}, \gamma_{i}$, be such that there exists $\alpha^{*}, t^{*}$ such that $\gamma\left(\alpha^{*}, t^{*}\right) \neq 0$. Let $S$ be the solution operator of the system (35)-(38). Then, for any Atwood ratio A, with $-1 \leq A<1$, and for any $t>t^{*}$, there does not exist a constant $M$ for all data $\dot{\mathbf{z}}, \dot{\gamma}_{i} \in H^{s^{\prime}}$ such that

$$
\left\|S\left[\dot{\mathbf{z}}, \dot{\gamma}_{i}\right]\left(\cdot, t, t^{\prime}\right)\right\|_{H^{s}} \leq M\left\|\left(\dot{\mathbf{z}}, \dot{\gamma}_{i}\right)\left(\cdot, t^{\prime}\right)\right\|_{H^{s^{\prime}}}
$$

for $t^{\prime} \leq t$ and $s \leq s^{\prime}$. This is ill-posedness in the sense of Hadamard.

The proof of the corollary is based on comparing the variable coefficient problem with a reduced frozen coefficient system using an argument due to Strang [24]. There is essentially no difference between the 2-D and the 3-D cases. In two space dimensions, this result has been proved by Beale-Hou-Lowengrub in [5].

Proof of Lemma 4.1. As we mentioned in the beginning of this section, we will first linearize the equations using the original Lagrangian variable, $\alpha$. By a direct calculation, we can easily obtain the linear variation of the velocity integral (20) as follows:

$$
\dot{\mathbf{U}}(\alpha, t)=\int\left(\dot{\mu}_{\alpha_{1}} \mathbf{z}_{\alpha_{2}}-\dot{\mu}_{\alpha_{2}} \mathbf{z}_{\alpha_{1}}\right) \times \nabla_{\mathbf{z}^{\prime}} G\left(\mathbf{z}(\alpha)-\mathbf{z}\left(\alpha^{\prime}\right)\right) d \alpha^{\prime}
$$




$$
\begin{aligned}
& +\int\left(\mu_{\alpha_{1}} \dot{\mathbf{z}}_{\alpha_{2}}-\mu_{\alpha_{2}} \dot{\mathbf{z}}_{\alpha_{1}}\right) \times \nabla_{\mathbf{z}^{\prime}} G\left(\mathbf{z}(\alpha)-\mathbf{z}\left(\alpha^{\prime}\right)\right) d \alpha^{\prime} \\
& -\int\left(\mu_{\alpha_{1}} \mathbf{z}_{\alpha_{2}}-\mu_{\alpha_{2}} \mathbf{z}_{\alpha_{1}}\right) \times\left(\nabla_{\mathbf{z}^{\prime}} \nabla_{\mathbf{z}^{\prime}} G\left(\mathbf{z}(\alpha)-\mathbf{z}\left(\alpha^{\prime}\right)\right) \cdot\left(\dot{\mathbf{z}}(\alpha)-\dot{\mathbf{z}}\left(\alpha^{\prime}\right)\right)\right) d \alpha^{\prime}
\end{aligned}
$$

The well-posedness of the linearized motion can be obtained using this Lagrangian frame, $\alpha$. However, because of the lack of orthogonality in the Lagrangian coordinate, the properties of the resulting singular operators become more complicated. This makes it more difficult to account for various cancellations among singular operators. To simplify the presentation, we will use an orthogonal parametrization of the unperturbed surface. This amounts to a change of variable from $\alpha$ to $\beta$ : $\alpha=\alpha(\beta, t)$, $\tilde{\mathbf{z}}(\beta, t)=\mathbf{z}(\alpha, t), \tilde{\mathbf{z}}(\beta, t)=\dot{\mathbf{z}}(\alpha, t)$, where $\beta$ is the orthogonal coordinates on the surface. Other quantities are defined similarly. We again emphasize that this change of variable is done after we obtain the linearized equations in the original Lagrangian variable, $\alpha$. By a direct calculation, we can show that (dropping the tildes)

$$
\begin{aligned}
\dot{\mathbf{U}}(\beta, t)= & \int\left(\dot{\mu}_{\beta_{1}} \mathbf{z}_{\beta_{2}}-\dot{\mu}_{\beta_{2}} \mathbf{z}_{\beta_{1}}\right) \times \nabla_{\mathbf{z}^{\prime}} G\left(\mathbf{z}(\beta)-\mathbf{z}\left(\beta^{\prime}\right)\right) d \beta^{\prime} \\
& +\int\left(\mu_{\beta_{1}} \dot{\mathbf{z}}_{\beta_{2}}-\mu_{\beta_{2}} \dot{\mathbf{z}}_{\beta_{1}}\right) \times \nabla_{\mathbf{z}^{\prime}} G\left(\mathbf{z}(\beta)-\mathbf{z}\left(\beta^{\prime}\right)\right) d \beta^{\prime} \\
& -\int\left(\mu_{\beta_{1}} \mathbf{z}_{\beta_{2}}-\mu_{\beta_{2}} \mathbf{z}_{\beta_{1}}\right) \times\left(\nabla_{\mathbf{z}^{\prime}} \nabla_{\mathbf{z}^{\prime}} G\left(\mathbf{z}(\beta)-\mathbf{z}\left(\beta^{\prime}\right)\right) \cdot\left(\dot{\mathbf{z}}(\beta)-\dot{\mathbf{z}}\left(\beta^{\prime}\right)\right)\right) d \beta^{\prime} .
\end{aligned}
$$

It is clear that the form of the linearized equations remains the same through this change of variable. But the properties of the underlining singular operators have changed due to this change of variable since we now work on an orthogonal coordinate $\beta$. On the other hand, this change of variable will bring in a new convection term due to the variation in the time derivative, as will be seen later.

We have derived the linearization for this velocity integral in our previous study of 3 -D water waves [16] in the orthogonal coordinates. The equations (35)-(37) can be obtained using the Lemma 4.1-4.3 in our 3-D water waves paper [16]. In fact, the problem is simpler here since the interface velocity is taken to be the average of the velocities above and below the interface. As a consequence, there is no contribution from the local term.

The following lemmas regarding the properties of the Hilbert transforms and the integral operator $K$ will be useful in obtaining our linearization. The proofs of these Lemmas can be found in our 3-D water wave paper [16].

LEMMA 4.2. (Commutator of the Hilbert Transform) Let $f$ be a smooth function. Then for any function $g$ in $H^{s}$, we have

$$
H_{l}(f g)=f H_{l}(g)+E_{-1}(g), \quad l=1,2 .
$$

That is, smooth functions can be passed in and out of the Hilbert transforms at the minor cost of introducing a lower order smoothing operator.

Lemma 4.3. (The Integral Operator $K$ ) Let $K$ be a double-layer potential operator in three dimensions defined in (17) with $\mathbf{z}$ evaluated at $\mathbf{z}(\alpha)$. We have $K f=$ $E_{-1}(f)$.

LEMma 4.4. (Properties of the Hilbert Transform)

(I) To the leading order, $\Lambda H_{\ell}$ is a local derivative operator:

$$
\Lambda H_{1}=-\left(\sigma_{1}\right)^{4}\left(\sigma_{2}\right)^{2} D_{1}+E_{0}
$$




$$
\Lambda H_{2}=-\left(\sigma_{1}\right)^{2}\left(\sigma_{2}\right)^{4} D_{2}+E_{0} .
$$

(II) The Hilbert transforms satisfy

$$
\left(\sigma_{1}\right)^{-2} H_{1}^{2}+\left(\sigma_{2}\right)^{-2} H_{2}^{2}=-\left(\sigma_{1} \sigma_{2}\right)^{2} I+E_{-1},
$$

and

$$
\left(\sigma_{1}\right)^{-2} H_{1} D_{2}=\left(\sigma_{2}\right)^{-2} H_{2} D_{1} .
$$

where $I$ is the identity operator.

Lemma 4.5. (The Essential Positivity of the $\Lambda$ Operator) The operator $\Lambda$ can be expressed as a sum of a first order positive operator and a bounded operator from $H^{s}$ to $H^{s}$.

The linearization for the vorticity equations (38) contains a number of terms. We will analyze them term by term. The first term is $\frac{\partial^{2} \mathbf{z}}{\partial t^{2}} \cdot \mathbf{z}_{\alpha_{i}}$ (here we omit the coefficient $-2 A)$. We have

$$
\left(\frac{\partial^{2} \mathbf{z}}{d t^{2}} \cdot \mathbf{z}_{\alpha_{i}}\right)^{\cdot}=\left(\frac{\partial \mathbf{U}}{d t} \cdot \mathbf{z}_{\alpha_{i}}\right)^{\cdot}=\frac{\partial \dot{\mathbf{U}}}{d t} \cdot \mathbf{z}_{\alpha_{i}}+\frac{\partial \mathbf{U}}{d t} \cdot \dot{\mathbf{z}}_{\alpha_{i}} \doteq \mathrm{I}_{1}+\mathrm{I}_{2} .
$$

The $I_{2}$ term is a lower order term. For the first term, $I_{1}$, we need to use the linearization result of $U$ in (35)-(37) and Lemma 4.4. Keeping only the leading order terms, we get

$$
\begin{aligned}
\mathrm{I}_{1}= & -\frac{1}{2}\left(\sigma_{1} \sigma_{2}\right)^{-1}\left(\gamma_{1} H_{1}+\gamma_{2} H_{2}\right) D_{i}\left(\dot{z}_{t}^{N}\right)+K_{i}\left(\frac{\partial \dot{\mu}}{\partial t}\right)+E_{0}\left(D_{l} \dot{\mathbf{z}}\right)+E_{0}(\dot{\gamma}) \\
= & -\frac{1}{4}\left\{\left(\sigma_{1} \sigma_{2}\right)^{-2}\left(\gamma_{1} H_{1}+\gamma_{2} H_{2}\right) D_{i}\left[H_{1}\left(\dot{\gamma}_{1}\right)+H_{2}\left(\dot{\gamma}_{2}\right)\right]\right. \\
& \left.+\sigma_{1} \gamma_{1}\left(\sigma_{1}^{2} \gamma_{1} D_{1}+\sigma_{2}^{2} \gamma_{2} D_{2}\right) D_{i}\left(\dot{z}^{T_{1}}\right)+\sigma_{2} \gamma_{2}\left(\sigma_{1}^{2} \gamma_{1} D_{1}+\sigma_{2}^{2} \gamma_{2} D_{2}\right) D_{i}\left(\dot{z}^{T_{2}}\right)\right\} \\
& +K_{i}\left(\frac{\partial \dot{\mu}}{\partial t}\right)+E_{0}\left(D_{l} \dot{\mathbf{z}}\right)+E_{0}(\dot{\gamma})
\end{aligned}
$$

where we have used $\tilde{\Lambda}=\left(\sigma_{1} \sigma_{2}\right)^{-2} \Lambda+E_{0}$ and Lemma 4.4 .

To estimate the second term, $\frac{1}{8}\left|\frac{\mathbf{W} \times \mathbf{N}}{J}\right|_{\alpha_{i}}^{2}$, we use the vector identity:

$$
\begin{aligned}
\left|\frac{\mathbf{W} \times \mathbf{N}}{J}\right|^{2} & =\frac{(\mathbf{W} \cdot \mathbf{W})(\mathbf{N} \cdot \mathbf{N})}{J^{2}}-\frac{(\mathbf{W} \cdot \mathbf{N})^{2}}{J^{2}} \\
& =\frac{\mathbf{W} \cdot \mathbf{W}}{J^{2}}-\frac{(\mathbf{W} \cdot \mathbf{N})^{2}}{J^{2}}
\end{aligned}
$$

Using $\mathbf{W} \cdot \mathbf{N}=0$, we can easily show that the variation of $(\mathbf{W} \cdot \mathbf{N})^{2} / J^{2}$ is identically zero. We have

$$
\frac{1}{8} D_{i}\left(\left|\frac{\mathbf{W} \times \mathbf{N}}{J}\right|^{2}\right)^{\cdot}=\frac{1}{4} D_{i}\left[\frac{\mathbf{W} \cdot \dot{\mathbf{W}}}{J^{2}}\right]-\frac{1}{4} D_{i}\left[\frac{(\mathbf{W} \cdot \mathbf{W})(\tilde{\mathbf{N}} \cdot \dot{\tilde{\mathbf{N}}})}{J^{4}}\right] \doteq \mathrm{I}_{3}+\mathrm{I}_{4},
$$

where $\tilde{\mathbf{N}}=\mathbf{z}_{\alpha_{1}} \times \mathbf{z}_{\alpha_{2}}$. By the definition of $\mathbf{W}$, we have

$$
\begin{aligned}
\mathrm{I}_{3}= & \frac{1}{4} D_{i}\left[\left(\sigma_{1} \sigma_{2}\right)^{2}\left(\gamma_{1} \mathbf{z}_{\alpha_{2}}-\gamma_{2} \mathbf{z}_{\alpha_{1}}\right) \cdot\left(\dot{\gamma}_{1} \mathbf{z}_{\alpha_{2}}+\gamma_{1} \dot{\mathbf{z}}_{\alpha_{2}}-\dot{\gamma}_{2} \mathbf{z}_{\alpha_{1}}-\gamma_{2} \dot{\mathbf{z}}_{\alpha_{1}}\right)\right] \\
= & \frac{1}{4} D_{i}\left[\sigma_{1}^{2} \gamma_{1} \dot{\gamma}_{1}+\sigma_{2}^{2} \gamma_{2} \dot{\gamma}_{2}+\sigma_{1} \sigma_{2}^{2} \gamma_{2}\left(\gamma_{2} D_{1}-\gamma_{1} D_{2}\right)\left(\dot{z}^{T_{1}}\right)\right. \\
& \left.-\sigma_{1}^{2} \sigma_{2} \gamma_{1}\left(\gamma_{2} D_{1}-\gamma_{1} D_{2}\right)\left(\dot{z}^{T_{2}}\right)\right]
\end{aligned}
$$


and

$$
\begin{aligned}
\mathrm{I}_{4} & =-D_{i}\left[\frac{\left(\sigma_{1} \sigma_{2}\right)^{3}}{4}(\mathbf{W} \cdot \mathbf{W})\left(\mathbf{N} \cdot\left(\dot{\mathbf{z}}_{\alpha_{1}} \times \mathbf{z}_{\alpha_{2}}+\mathbf{z}_{\alpha_{1}} \times \dot{\mathbf{z}}_{\alpha_{2}}\right)\right)\right] \\
& =-\frac{1}{4}\left(\sigma_{1}^{2} \gamma_{1}^{2}+\sigma_{2}^{2} \gamma_{2}^{2}\right) D_{i}\left[\sigma_{1} D_{1}\left(\dot{z}^{T_{1}}\right)+\sigma_{2} D_{2}\left(\dot{z}^{T_{2}}\right)\right]+E_{0}\left(D_{l} \dot{\mathbf{z}}\right) .
\end{aligned}
$$

The third term, $\mathbf{g} \cdot \mathbf{z}_{\alpha_{i}}$, only contributes to lower order terms. The fourth term is about the surface tension, $\tau \kappa_{\alpha_{i}}$. The mean curvature is defined by

$$
\begin{aligned}
\kappa & =\frac{1}{2}\left(\frac{\left|\mathbf{z}_{\alpha_{1}}\right|^{2}\left(\mathbf{N} \cdot \mathbf{z}_{\alpha_{2} \alpha_{2}}\right)-2\left(\mathbf{z}_{\alpha_{1}} \cdot \mathbf{z}_{\alpha_{2}}\right)\left(\mathbf{N} \cdot \mathbf{z}_{\alpha_{1} \alpha_{2}}\right)+\left|\mathbf{z}_{\alpha_{2}}\right|^{2}\left(\mathbf{N} \cdot \mathbf{z}_{\alpha_{1} \alpha_{1}}\right)}{\left|\mathbf{z}_{\alpha_{1}} \times \mathbf{z}_{\alpha_{2}}\right|^{2}}\right) \\
& \left.=\frac{1}{2}\left(\sigma_{1}\left(\mathbf{T}_{1}\right)_{\alpha_{1}} \cdot \mathbf{N}+\sigma_{2}\left(\mathbf{T}_{2}\right)_{\alpha_{2}} \cdot \mathbf{N}\right) \frac{\left|\mathbf{z}_{\alpha_{1}}\right|^{2}\left|\mathbf{z}_{\alpha_{2}}\right|^{2}}{\left|\mathbf{z}_{\alpha_{1}} \times \mathbf{z}_{\alpha_{2}}\right|^{2}}-2 \frac{\left(\mathbf{z}_{\alpha_{1}} \cdot \mathbf{z}_{\alpha_{2}}\right)\left(\mathbf{N} \cdot \mathbf{z}_{\alpha_{1} \alpha_{2}}\right)}{\left|\mathbf{z}_{\alpha_{1}} \times \mathbf{z}_{\alpha_{2}}\right|^{2}}\right) .
\end{aligned}
$$

It is important to realize that although $\mathbf{z}_{\alpha_{1}}$ is orthogonal to $\mathbf{z}_{\alpha_{2}}$, the corresponding perturbed vectors do not necessarily have this property. For this reason, it is important to use the definition of curvature for general coordinates, and perform variation for all terms. The orthogonality of the unperturbed smooth tangent vectors can be used only after the linearization.

First, we can show by direct calculations that

$$
\left(\frac{\left|\mathbf{z}_{\alpha_{1}}\right|^{2}\left|\mathbf{z}_{\alpha_{2}}\right|^{2}}{\left|\mathbf{z}_{\alpha_{1}} \times \mathbf{z}_{\alpha_{2}}\right|^{2}}\right)^{\cdot}=0
$$

by using the orthogonality condition after the linearization. With this observation, the variation of curvature can be proceeded as follows:

$$
\begin{aligned}
\dot{\kappa} & =\frac{1}{2}\left\{\left(\dot{\sigma}_{1}\left(\mathbf{T}_{1}\right)_{\alpha_{1}} \cdot \mathbf{N}+\dot{\sigma}_{2}\left(\mathbf{T}_{2}\right)_{\alpha_{2}} \cdot \mathbf{N}\right)+\left(\sigma_{1}\left(\dot{\mathbf{T}}_{1}\right)_{\alpha_{1}} \cdot \mathbf{N}+\sigma_{2}\left(\dot{\mathbf{T}}_{2}\right)_{\alpha_{2}} \cdot \mathbf{N}\right)\right. \\
& \left.+\sigma_{1}\left(\mathbf{T}_{1}\right)_{\alpha_{1}} \cdot \dot{\mathbf{N}}+\sigma_{2}\left(\mathbf{T}_{2}\right)_{\alpha_{2}} \cdot \dot{\mathbf{N}}-2 \frac{\dot{\mathbf{z}}_{\alpha_{1}} \cdot \mathbf{z}_{\alpha_{2}}+\mathbf{z}_{\alpha_{1}} \cdot \dot{\mathbf{z}}_{\alpha_{2}}}{\left|\mathbf{z}_{\alpha_{1}} \times \mathbf{z}_{\alpha_{2}}\right|^{2}}\left(\mathbf{N} \cdot \mathbf{z}_{\alpha_{1} \alpha_{2}}\right)\right\} \\
& =\frac{1}{2}\left(\dot{\sigma}_{1}\left(\mathbf{T}_{1}\right)_{\alpha_{1}} \cdot \mathbf{N}+\dot{\sigma}_{2}\left(\mathbf{T}_{2}\right)_{\alpha_{2}} \cdot \mathbf{N}\right) \\
& +\frac{1}{2}\left\{\left(\sigma_{1}\left(\dot{\mathbf{T}}_{1} \cdot \mathbf{N}\right)_{\alpha_{1}}+\sigma_{2}\left(\dot{\mathbf{T}}_{2} \cdot \mathbf{N}\right)_{\alpha_{2}}\right)-\left(\sigma_{1} \dot{\mathbf{T}}_{1} \cdot \mathbf{N}_{\alpha_{1}}+\sigma_{2} \dot{\mathbf{T}}_{2} \cdot \mathbf{N}_{\alpha_{2}}\right)\right\} \\
& +\frac{1}{2}\left\{\sigma_{1}\left(\mathbf{T}_{1}\right)_{\alpha_{1}} \cdot \dot{\mathbf{N}}+\sigma_{2}\left(\mathbf{T}_{2}\right)_{\alpha_{2}} \cdot \dot{\mathbf{N}}-2 \frac{\dot{\mathbf{z}}_{\alpha_{1}} \cdot \mathbf{z}_{\alpha_{2}}+\mathbf{z}_{\alpha_{1}} \cdot \dot{\mathbf{z}}_{\alpha_{2}}}{\left|\mathbf{z} \cdot \mathbf{z}_{\alpha_{1}} \times \mathbf{z}_{\alpha_{2}}\right|^{2}}(\mathbf{N})\right\}
\end{aligned}
$$

We define

$$
\left(\mathbf{T}_{1}\right)_{\alpha_{i}}=\Gamma_{i}^{1} \mathbf{N}+\Gamma_{i}^{12} \mathbf{T}_{2}, \quad\left(\mathbf{T}_{2}\right)_{\alpha_{i}}=\Gamma_{i}^{2} \mathbf{N}+\Gamma_{i}^{21} \mathbf{T}_{1}
$$

It follows that

$$
\mathbf{N}_{\alpha_{i}}=-\Gamma_{i}^{1} \mathbf{T}_{1}-\Gamma_{i}^{2} \mathbf{T}_{2}, \quad \Gamma_{i}^{12}=-\Gamma_{i}^{21}
$$

We claim that

$$
\sigma_{1} \Gamma_{1}^{2}=\sigma_{2} \Gamma_{2}^{1}
$$

This can be proved as follows

$$
\begin{aligned}
\sigma_{1} \Gamma_{1}^{2} & =\sigma_{1}\left(\mathbf{T}_{2}\right)_{\alpha_{1}} \cdot \mathbf{N}=\sigma_{1}\left(\sigma_{2} \mathbf{z}_{\alpha_{2}}\right)_{\alpha_{1}} \cdot \mathbf{N} \\
& =\left(\sigma_{1} \sigma_{2}\right) \mathbf{z}_{\alpha_{1} \alpha_{2}} \cdot \mathbf{N}=\sigma_{2} \Gamma_{2}^{1}
\end{aligned}
$$


To find $\dot{\sigma}_{i}$, we use the identity $\sigma_{i}^{-2}=\mathbf{z}_{\alpha_{i}} \cdot \mathbf{z}_{\alpha_{i}}$. We obtain

$$
-\sigma_{i}^{-3} \dot{\sigma}_{i}=\mathbf{z}_{\alpha_{i}} \cdot \dot{\mathbf{z}}_{\alpha_{i}}=\sigma_{i}^{-1} D_{i} \dot{z}^{T_{i}}+E_{0}(\dot{\mathbf{z}})
$$

and

$$
\begin{aligned}
\dot{\mathbf{T}}_{i} \cdot \mathbf{N} & =\sigma_{i} \dot{\mathbf{z}}_{\alpha_{i}} \cdot \mathbf{N}=\sigma_{i} D_{i} \dot{z}^{N}-\sigma_{i} \dot{\mathbf{z}} \cdot \mathbf{N}_{\alpha_{i}} \\
& =\sigma_{i} D_{i} \dot{z}^{N}+\sigma_{i} \Gamma_{i}^{1} \dot{z}^{T_{1}}+\sigma_{i} \Gamma_{i}^{2} \dot{z}^{T_{2}} .
\end{aligned}
$$

Using (51)-(53) and $\dot{\mathbf{T}}_{i} \cdot \mathbf{T}_{i}=0$, we can show that

$$
\frac{\dot{\mathbf{z}}_{\alpha_{1}} \cdot \mathbf{z}_{\alpha_{2}}+\mathbf{z}_{\alpha_{1}} \cdot \dot{\mathbf{z}}_{\alpha_{2}}}{\left|\mathbf{z}_{\alpha_{1}} \times \mathbf{z}_{\alpha_{2}}\right|^{2}}\left(\mathbf{N} \cdot \mathbf{z}_{\alpha_{1} \alpha_{2}}\right)=\sigma_{1}^{2} \Gamma_{1}^{2} \dot{z}_{\alpha_{1}}^{T_{2}}+\sigma_{2}^{2} \Gamma_{2}^{1} \dot{z}_{\alpha_{2}}^{T_{1}}+E_{0}(\dot{\mathbf{z}})
$$

and

$$
\sigma_{1}\left(\mathbf{T}_{1}\right)_{\alpha_{1}} \cdot \dot{\mathbf{N}}+\sigma_{2}\left(\mathbf{T}_{2}\right)_{\alpha_{2}} \cdot \dot{\mathbf{N}}=-\sigma_{1} \sigma_{2}\left(\Gamma_{1}^{12} \dot{z}_{\alpha_{2}}^{N}+\Gamma_{2}^{21} \dot{z}_{\alpha_{1}}^{N}\right)+E_{0}(\dot{\mathbf{z}}) .
$$

Substituting (54)-(58) into (50), we find that all the tangential derivative terms cancel one another. Only the normal variation terms survive to the leading order. Thus we obtain the variation of mean curvature as follows:

(59) $\dot{\kappa}=\frac{1}{2}\left[\sigma_{1} D_{1}\left(\sigma_{1} D_{1} \dot{z}^{N}\right)+\sigma_{2} D_{2}\left(\sigma_{2} D_{2} \dot{z}^{N}\right)\right]-\frac{1}{2} \sigma_{1} \sigma_{2}\left(\Gamma_{1}^{12} \dot{z}_{\alpha_{2}}^{N}+\Gamma_{2}^{21} \dot{z}_{\alpha_{1}}^{N}\right)+E_{0}(\dot{\mathbf{z}})$.

Combining the variation results for the first term, the second term and the fourth term, we complete our derivation of the linearized equation for the vorticity equation (24).

Finally, we remark that the left hand side of (24) can be easily derived by using chain rule:

$$
\frac{\partial \dot{z}(\alpha, t)}{\partial t}=\frac{\partial \tilde{\dot{z}}(\beta, t)}{\partial t}-\left(\frac{\partial \alpha(\beta, t)}{\partial t} Q^{-1}\right) \cdot \nabla_{\beta} \tilde{\dot{z}}=\frac{D \tilde{\dot{z}}}{D t}
$$

This completes the derivation of our linearized equations.

We remark that the variation of mean curvature is of interest by itself. This has been studied by other people in literature. In particular, we would like to mention a related result by Brower et al. [7] in which they derived the normal variation of the mean curvature in general coordinates.

5. Energy estimates and the well-posedness analysis. In this section, we analyze the well-posedness of the linearized equations (35)-(38) by performing careful energy estimates. The special case of $A=0$ corresponds to the vortex sheet problem with surface tension. This is the most singular case in some sense and it represents the essential difficulty in the energy estimates for the general case. To simplify the presentation of the analysis, we first present the proof of Theorem 3.1 in the case of $A=0$. The main idea of our analysis for the case $A=0$ can be used to analyze the general case. The additional terms in the $\gamma$ equation can be handled similarly.

Proof of Theorem 3.1. Case I. $A=0$. 
The analysis is based on the general framework developed in [3] and [16]. The projection of the linearized variables into the tangent and normal coordinates is an essential step. This helps identify the leading order structures of the linearized system, and enables us to look for the right balance among various singular operators. To make the presentation easier to follow, we will divide the energy estimates into the several steps.

\section{Step 1. Simplification of leading order equations via change of variables.}

Before we perform energy estimates for the perturbed quantities, we would like to simplify the equations for the perturbed quantities by change of variables. The balance among various terms becomes more apparent under this new set of variables.

First, we would like to eliminate one of the tangential variables so that we obtain a system qualitatively similar to the 2 -D case $[5,6]$. To this end, we introduce the new tangential variables $\dot{\phi}_{1}$ and $\dot{\phi}_{2}$ as follows:

$$
\begin{aligned}
& \dot{\phi}_{1}=\left(\sigma_{2}\right)^{-1} H_{2} \dot{z}^{T_{1}}-\left(\sigma_{1}\right)^{-1} H_{1} \dot{z}^{T_{2}} \\
& \dot{\phi}_{2}=\left(\sigma_{1}\right)^{-1} H_{1} \dot{z}^{T_{1}}+\left(\sigma_{2}\right)^{-1} H_{2} \dot{z}^{T_{2}} .
\end{aligned}
$$

By Lemma 4.4, we have

$$
\begin{aligned}
& \dot{z}^{T_{1}}=-\left(\sigma_{1} \sigma_{2}\right)^{2}\left[\left(\sigma_{2}\right)^{-1} H_{2} \dot{\phi}_{1}+\left(\sigma_{1}\right)^{-1} H_{1} \dot{\phi}_{2}\right]+E_{-1}(\dot{\mathbf{z}}), \\
& \dot{z}^{T_{2}}=\left(\sigma_{1} \sigma_{2}\right)^{2}\left[\left(\sigma_{1}\right)^{-1} H_{1} \dot{\phi}_{1}-\left(\sigma_{2}\right)^{-1} H_{2} \dot{\phi}_{2}\right]+E_{-1}(\dot{\mathbf{z}}) .
\end{aligned}
$$

Recall that

$$
\frac{D \dot{z}}{D t}=\frac{\partial \dot{z}}{\partial t}-\left(\frac{\partial \alpha(\beta, t)}{\partial t} Q^{-1}\right) \cdot \nabla_{\beta} \dot{z}
$$

It is important to note that $\left(\frac{\partial \alpha(\beta, t)}{\partial t} Q^{-1}\right)$ depends on $\mathbf{z}(\alpha, t)$ only, not on the perturbed quantity $\dot{\mathbf{z}}$. Thus $\frac{D}{D t}$ is a linear operator to the leading order. In terms of these new variables, Eqs. (35)-(38) become

$$
\begin{aligned}
\frac{D \dot{\phi}_{1}}{D t}= & E_{0}(\dot{\phi})+E_{0}\left(\dot{z}^{N}\right)+\widetilde{K}_{1}(\dot{\mu}) \\
\frac{D \dot{\phi}_{2}}{D t}= & -\frac{1}{2} \sigma_{1} \sigma_{2} \widetilde{\Lambda}\left(\gamma_{1} H_{1}+\gamma_{2} H_{2}\right) \dot{z}^{N}+E_{0}(\dot{\phi})+E_{0}\left(\dot{z}^{N}\right)+\widetilde{K}_{2}(\dot{\mu}) \\
\frac{D \dot{z}^{N}}{D t}= & \frac{1}{2} \sigma_{1} \sigma_{2} \widetilde{\Lambda}(\dot{\mu})+\frac{1}{2}\left(\sigma_{1} \sigma_{2}\right)^{2} \widetilde{\Lambda}\left[\left(\sigma_{1}\right)^{2} \gamma_{1} H_{2}-\left(\sigma_{2}\right)^{2} \gamma_{2} H_{1}\right] \dot{\phi}_{1} \\
& +\frac{1}{2}\left(\sigma_{1} \sigma_{2}\right)^{3} \widetilde{\Lambda}\left(\gamma_{1} H_{1}+\gamma_{2} H_{2}\right) \dot{\phi}_{2}+E_{0}(\dot{\phi})+E_{0}\left(\dot{z}^{N}\right) \\
\frac{D \dot{\gamma}_{l}}{D t}= & \frac{\tau}{2} D_{l}\left\{\sigma_{1} D_{1}\left(\sigma_{1} D_{1} \dot{z}^{N}\right)+\sigma_{2} D_{2}\left(\sigma_{2} D_{2} \dot{z}^{N}\right)\right. \\
& \left.-\sigma_{1} \sigma_{2}\left(\Gamma_{1}^{12} \dot{z}_{\alpha_{2}}^{N}+\Gamma_{2}^{21} \dot{z}_{\alpha_{1}}^{N}\right)\right\}+E_{0}\left(D_{l} \dot{z}\right)+E_{0}(\dot{\gamma})
\end{aligned}
$$

where $\widetilde{K}_{1}=\left(\sigma_{2}\right)^{-1} H_{2} K_{1}-\left(\sigma_{1}\right)^{-1} H_{1} K_{2}$, and $\widetilde{K}_{2}=\left(\sigma_{1}\right)^{-1} H_{1} K_{1}+\left(\sigma_{2}\right)^{-1} H_{2} K_{2}$.

Note that the equations for the interface variables, $\dot{\mathbf{z}}$, are coupled to the $\dot{\gamma}_{1}$ and $\dot{\gamma}_{2}$ variables through the $\dot{\mu}$ variable. This is consistent since $\gamma_{1}$ and $\gamma_{2}$ are obtained from the $\mu$ variable by $\gamma_{l}=D_{l} \mu$. Thus it makes sense to introduce $\dot{\mu}$ as the new variable instead of using $\dot{\gamma}_{1}$ and $\dot{\gamma}_{2}$. This also reduces the total number of equations 
by one. We have

$$
\begin{aligned}
\frac{D \dot{\phi}_{1}}{D t}= & E_{0}(\dot{\phi})+E_{0}\left(\dot{z}^{N}\right)+\widetilde{K}_{1}(\dot{\mu}) \\
\frac{D \dot{\phi}_{2}}{D t}= & -\frac{1}{2} \sigma_{1} \sigma_{2} \widetilde{\Lambda}\left(\gamma_{1} H_{1}+\gamma_{2} H_{2}\right) \dot{z}^{N}+E_{0}(\dot{\phi})+E_{0}\left(\dot{z}^{N}\right)+\widetilde{K}_{2}(\dot{\mu}) \\
\frac{D \dot{z}^{N}}{D t}= & \frac{1}{2} \sigma_{1} \sigma_{2} \widetilde{\Lambda}(\dot{\mu})+\frac{1}{2}\left(\sigma_{1} \sigma_{2}\right)^{2} \widetilde{\Lambda}\left[\left(\sigma_{1}\right)^{2} \gamma_{1} H_{2}-\left(\sigma_{2}\right)^{2} \gamma_{2} H_{1}\right] \dot{\phi}_{1} \\
& +\frac{1}{2}\left(\sigma_{1} \sigma_{2}\right)^{3} \widetilde{\Lambda}\left(\gamma_{1} H_{1}+\gamma_{2} H_{2}\right) \dot{\phi}_{2}+E_{0}(\dot{\phi})+E_{0}\left(\dot{z}^{N}\right) \\
\frac{D \dot{\mu}}{D t}= & \frac{\tau}{2}\left\{\sigma_{1} D_{1}\left(\sigma_{1} D_{1} \dot{z}^{N}\right)+\sigma_{2} D_{2}\left(\sigma_{2} D_{2} \dot{z}^{N}\right)\right. \\
& \left.-\sigma_{1} \sigma_{2}\left(\Gamma_{1}^{12} \dot{z}_{\alpha_{2}}^{N}+\Gamma_{2}^{21} \dot{z}_{\alpha_{1}}^{N}\right)\right\}+E_{0}(\dot{z})+E_{0}(\dot{\mu}) .
\end{aligned}
$$

On the other hand, we note that it is through the coupling of Eq. (61) and Eq. (62) that introduces the Kelvin-Helmholtz instability. If it were not for the coupling of the $\dot{z}$ equation to the $\dot{\mu}$ equation, the problem would have been ill-posed due to the Kelvin-Helmholtz instability. In order to see clearly how the surface tension effect stabilizes the Kelvin-Helmholtz instability, we would like to bring these two competing stability mechanisms into the same equation. For this purpose, we incorporate other tangential terms into our modified $\dot{\mu}$ variable, which we denote by $\dot{\Gamma}$. Specifically, our new $\dot{\Gamma}$ variable is defined as follows:

$$
\dot{\Gamma}=-\dot{\mu}+\left(\sigma_{1} \sigma_{2}\right)\left[\left(\sigma_{2}\right)^{2} \gamma_{2} H_{1}-\left(\sigma_{1}\right)^{2} \gamma_{1} H_{2}\right] \dot{\phi}_{1}-2\left(\sigma_{1} \sigma_{2}\right)^{2}\left(\gamma_{1} H_{1}+\gamma_{2} H_{2}\right) \dot{\phi}_{2} .
$$

In terms of the $\dot{\Gamma}$ variable, we have

$$
\begin{aligned}
\frac{D \dot{\phi}_{1}}{D t}= & E_{0}(\dot{\phi})+E_{0}(\dot{\mathbf{z}})+\widetilde{K}_{1}(\dot{\mu}) \\
\frac{D \dot{\phi}_{2}}{D t}= & -\frac{1}{2} \sigma_{1} \sigma_{2} \widetilde{\Lambda}\left(\gamma_{1} H_{1}+\gamma_{2} H_{2}\right) \dot{z}^{N}+E_{0}(\dot{\phi})+E_{0}(\dot{\mathbf{z}})+\widetilde{K}_{2}(\dot{\mu}) \\
\frac{D \dot{z}^{N}}{D t}= & -\frac{1}{2} \widetilde{\Lambda} \dot{\Gamma}-\frac{1}{2}\left(\sigma_{1} \sigma_{2}\right)^{3} \widetilde{\Lambda}\left(\gamma_{1} H_{1}+\gamma_{2} H_{2}\right) \dot{\phi}_{2}+E_{0}(\dot{\phi})+E_{0}\left(\dot{z}^{N}\right) \\
\frac{D \dot{\Gamma}}{D t}= & -\frac{\tau}{2}\left\{\sigma_{1} D_{1}\left(\sigma_{1} D_{1} \dot{z}^{N}\right)+\sigma_{2} D_{2}\left(\sigma_{2} D_{2} \dot{z}^{N}\right)-\sigma_{1} \sigma_{2}\left(\Gamma_{1}^{12} \dot{z}_{\alpha_{2}}^{N}+\Gamma_{2}^{21} \dot{z}_{\alpha_{1}}^{N}\right)\right\} \\
& +\left(\sigma_{1} \sigma_{2}\right)\left(\gamma_{1} H_{1}+\gamma_{2} H_{2}\right) \Lambda\left(\gamma_{1} H_{1}+\gamma_{2} H_{2}\right) \dot{z}^{N}+E_{0}(\dot{\phi})+E_{0}(\dot{\mathbf{z}})+E_{0}(\dot{\Gamma}) .
\end{aligned}
$$

Note the change of sign in the $\dot{\phi}_{2}$ term in the $\frac{D \dot{z}^{N}}{D t}$ equation as a consequence of this change of variables. This in effect changes the coupling between $\dot{\phi}_{2}$ and $\dot{z}^{N}$ from elliptic ( i.e. the Kelvin-Helmholtz instability) to hyperbolic. The effect of the Kelvin-Helmholtz instability is now shifted to the last $\dot{z}^{N}$ term in the $\frac{D \dot{\Gamma}}{D t}$ equation.

We define a new operator, $\mathcal{L}$, as follows

$$
\begin{aligned}
\mathcal{L}= & -\frac{\tau}{2}\left\{\left(\sigma_{2}\right)^{-1} D_{1}\left(\sigma_{1} D_{1}\right)+\left(\sigma_{1}\right)^{-1} D_{2}\left(\sigma_{2} D_{2}\right)\right. \\
& \left.-\left(\Gamma_{1}^{12} D_{2}+\Gamma_{2}^{21} D_{1}\right)\right\}+\left(\gamma_{1} H_{1}+\gamma_{2} H_{2}\right) \Lambda\left(\gamma_{1} H_{1}+\gamma_{2} H_{2}\right) .
\end{aligned}
$$

Recall that

$$
\frac{D \dot{z}}{D t}=\frac{\partial \dot{z}}{\partial t}-\left(\frac{\partial \alpha(\beta, t)}{\partial t} Q^{-1}\right) \cdot \nabla_{\beta} \dot{z}
$$


We denote $\left(\frac{\partial \alpha(\beta, t)}{\partial t} Q^{-1}\right)$ by $\mathbf{w}(\alpha, t)$ to simplify the notation. As we mentioned before, $\mathbf{w}$ depends on $\mathbf{z}$ only, not on $\dot{\mathbf{z}}$. Moving the convection terms to the right hand side of the equations, we have

(65) $\frac{\partial \dot{\phi}_{1}}{\partial t}=\mathbf{w} \cdot \nabla \dot{\phi}_{1}+E_{0}(\dot{\phi})+E_{0}(\dot{\mathbf{z}})+\widetilde{K}_{1}(\dot{\mu})$

(66) $\frac{\partial \dot{\phi}_{2}}{\partial t}=-\frac{1}{2} \sigma_{1} \sigma_{2} \widetilde{\Lambda}\left(\gamma_{1} H_{1}+\gamma_{2} H_{2}\right) \dot{z}^{N}+\mathbf{w} \cdot \nabla \dot{\phi}_{2}+E_{0}(\dot{\phi})+E_{0}(\dot{\mathbf{z}})+\widetilde{K}_{2}(\dot{\mu})$

(67) $\frac{\partial \dot{z}^{N}}{\partial t}=-\frac{1}{2} \sigma_{1} \sigma_{2} \tilde{\Lambda} \dot{\Gamma}-\frac{1}{2}\left(\sigma_{1} \sigma_{2}\right)^{3} \widetilde{\Lambda}\left(\gamma_{1} H_{1}+\gamma_{2} H_{2}\right) \dot{\phi}_{2}+\mathbf{w} \cdot \nabla \dot{z}^{N}+E_{0}(\dot{\phi})+E_{0}\left(\dot{z}^{N}\right)$

$$
\frac{\partial \dot{\Gamma}}{\partial t}=\sigma_{1} \sigma_{2} \mathcal{L} \dot{z}^{N}+\mathbf{w} \cdot \nabla \dot{\Gamma}+E_{0}(\dot{\phi})+E_{0}(\dot{\mathbf{z}})+E_{0}(\dot{\Gamma})
$$

To make it clear how we do our energy estimates, we further decompose the right hand sides of the above equations into the leading order terms and the lower order terms. More precisely, we denote the lower order terms by

$$
\begin{aligned}
& R_{1}=\mathbf{w} \cdot \nabla \dot{\phi}_{1}+E_{0}(\dot{\phi})+E_{0}(\dot{\mathbf{z}})+\widetilde{K}_{1}(\dot{\mu}) \\
& R_{2}=\mathbf{w} \cdot \nabla \dot{\phi}_{2}+E_{0}(\dot{\phi})+E_{0}(\dot{\mathbf{z}})+\widetilde{K}_{2}(\dot{\mu}) \\
& R_{3}=\mathbf{w} \cdot \nabla \dot{z}^{N}+E_{0}(\dot{\phi})+E_{0}\left(\dot{z}^{N}\right) \\
& R_{4}=\mathbf{w} \cdot \nabla \dot{\Gamma}+E_{0}(\dot{\phi})+E_{0}(\dot{\mathbf{z}})+E_{0}(\dot{\Gamma}) .
\end{aligned}
$$

Then the evolution equations for the perturbed quantities become

$$
\begin{aligned}
\frac{\partial \dot{\phi}_{1}}{\partial t} & =R_{1} \\
\frac{\partial \dot{\phi}_{2}}{\partial t} & =-\frac{1}{2} \sigma_{1} \sigma_{2} \widetilde{\Lambda}\left(\gamma_{1} H_{1}+\gamma_{2} H_{2}\right) \dot{z}^{N}+R_{2} \\
\frac{\partial \dot{z}^{N}}{\partial t} & =-\frac{1}{2} \sigma_{1} \sigma_{2} \widetilde{\Lambda} \dot{\Gamma}-\frac{1}{2}\left(\sigma_{1} \sigma_{2}\right)^{3} \widetilde{\Lambda}\left(\gamma_{1} H_{1}+\gamma_{2} H_{2}\right) \dot{\phi}_{2}+R_{3} \\
\frac{\partial \dot{\Gamma}}{\partial t} & =\sigma_{1} \sigma_{2} \mathcal{L} \dot{z}^{N}+R_{4}
\end{aligned}
$$

\section{Step 2. Energy estimate for the leading order terms.}

We will first derive energy estimates for the leading order terms by using the $H^{1}$ norm for $\dot{\phi}_{i}(i=1,2)$ and $\dot{z}^{N}$, and using the $H^{1 / 2}$ norm for $\dot{\Gamma}$. The strategy is follows. The main balance between the Kelvin-Helmholtz instability and the surface tension stabilizing effect is through the coupling of the $\dot{z}^{N}$ equation and the $\dot{\Gamma}$ equation. If we multiply $2 \mathcal{L} \dot{z}^{N}$ to the $\dot{z}^{N}$ equation, and multiply $\tilde{\Lambda} \dot{\Gamma}$ to the $\dot{\Gamma}$ equation, and add the resulting equations, the leading order terms cancel each other. The coupling between the second term in the $\dot{z}^{N}$ equation and the leading order term in the $\dot{\phi}_{2}$ equation is hyperbolic. By multiplying $2 \mathcal{L} \dot{\phi}_{2}$ to the $\dot{\phi}_{2}$ equation and adding it to the modified $\dot{z}^{N}$ equation, then all the leading order terms cancel each other after integration by parts. Only lower order terms remain.

Before we carry out the actual energy estimate for the leading order terms, we first prove an important property of the operators, $\mathcal{L}$ and $\tilde{\Lambda}$.

LEMMA 5.1. $\mathcal{L}$ is self adjoint up to a bounded operator. That is, for any function $f$ in $H^{s}$, we have

$$
\mathcal{L}^{*} f=\mathcal{L} f+E_{0}(f)
$$


Proof. Using Lemma 4.2 and the definition of $\Lambda$ and $H_{i}$, one easily show that $\Lambda^{*}=\Lambda+E_{0}$ and $H_{i}^{*}=-H_{i}+E_{-1}$. Thus the second term in $\mathcal{L}$ is self-adjoint up to a bounded operator. For the first term in $\mathcal{L}$, a direct calculation gives

$$
\begin{aligned}
\mathcal{L}^{*} f-\mathcal{L} f & =\tau\left[\sigma_{1} D_{1}\left(\left(\sigma_{2}\right)^{-1}\right) D_{1} f+\sigma_{2} D_{2}\left(\left(\sigma_{1}\right)^{-1}\right) D_{2} f+\Gamma_{1}^{12} D_{2} f+\Gamma_{2}^{21} D_{1} f\right]+E_{0}(f) \\
& =\tau\left[\left(\sigma_{1} D_{1}\left(\left(\sigma_{2}\right)^{-1}\right)+\Gamma_{2}^{21}\right) D_{1} f+\left(\sigma_{2} D_{2}\left(\left(\sigma_{1}\right)^{-1}\right)+\Gamma_{1}^{12}\right) D_{2} f\right]+E_{0}(f) .
\end{aligned}
$$

Recall that $\left(\sigma_{2}^{-1}\right)^{2}=\mathbf{z}_{\alpha_{2}} \cdot \mathbf{z}_{\alpha_{2}}$, and $\Gamma_{2}^{21}=\mathbf{T}_{\alpha_{2}} \cdot \mathbf{T}_{\alpha_{1}}=\left(\sigma_{2} \mathbf{z}_{\alpha_{2}}\right)_{\alpha_{2}} \cdot\left(\sigma_{1} \mathbf{z}_{\alpha_{1}}\right)$. Thus we obtain by using the orthogonality condition, $\mathbf{z}_{\alpha_{1}} \cdot \mathbf{z}_{\alpha_{2}}=0$,

$$
\begin{aligned}
\sigma_{1} D_{1}\left(\sigma_{2}^{-1}\right)+\Gamma_{2}^{21} & =\sigma_{1} \sigma_{2}\left(\mathbf{z}_{\alpha_{1} \alpha_{2}} \cdot \mathbf{z}_{\alpha_{2}}+\mathbf{z}_{\alpha_{2} \alpha_{2}} \cdot \mathbf{z}_{\alpha_{1}}\right) \\
& =\sigma_{1} \sigma_{2}\left(\mathbf{z}_{\alpha_{1}} \cdot \mathbf{z}_{\alpha_{2}}\right)_{\alpha_{2}}=0 .
\end{aligned}
$$

Similarly, we can show $\sigma_{2} D_{2}\left(\sigma_{1}^{-1}\right)+\Gamma_{1}^{12}=0$. This implies that $\mathcal{L}^{*} f=\mathcal{L} f+E_{0}(f)$.

LEMMA 5.2. $\widetilde{\Lambda}$ is a self-adjoint operator

$$
\widetilde{\Lambda}^{*}=\widetilde{\Lambda}
$$

Proof. By the definition of $\widetilde{\Lambda}$, we obtain by integration by parts

$$
\begin{aligned}
\widetilde{\Lambda} f & =\mathbf{z}_{\alpha_{1}} \times \mathbf{z}_{\alpha_{2}} \cdot \int\left(D_{1} f \mathbf{z}_{\alpha_{2}}-D_{2} f \mathbf{z}_{\alpha_{1}}\right) \times \nabla_{z^{\prime}} G\left(\mathbf{z}(\alpha)-\mathbf{z}\left(\alpha^{\prime}\right)\right) d \alpha^{\prime} \\
& =\int f\left(\alpha^{\prime}\right) \mathbf{z}_{\alpha_{1}} \times \mathbf{z}_{\alpha_{2}}(\alpha) \cdot \nabla_{z^{\prime}} \nabla_{z^{\prime}} G\left(\mathbf{z}(\alpha)-\mathbf{z}\left(\alpha^{\prime}\right)\right) \cdot\left(\mathbf{z}_{\alpha_{1}} \times \mathbf{z}_{\alpha_{1}}\right)\left(\alpha^{\prime}\right) d \alpha^{\prime} .
\end{aligned}
$$

Now it is clear that $\widetilde{\Lambda}$ is self-adjoint since the kernel remains the same by exchanging $\mathbf{z}(\alpha)$ with $\mathbf{z}\left(\alpha^{\prime}\right)$. This completes the proof of Lemma 5.2.

We now begin our energy estimate for the leading order terms. We multiply by $2 \mathcal{L} \dot{z}^{N}$ to the $\dot{z}^{N}$ equation, and integrate with respect to $\alpha$. This gives

$$
\begin{aligned}
& \frac{d}{d t}\left(\dot{z}^{N}, \mathcal{L} \dot{z}^{N}\right) \\
= & -\left(\sigma_{1} \sigma_{2} \widetilde{\Lambda} \dot{\Gamma}, \mathcal{L} \dot{z}^{N}\right)-\left(\left(\sigma_{1} \sigma_{2}\right)^{3} \widetilde{\Lambda}\left(\gamma_{1} H_{1}+\gamma_{2} H_{2}\right) \dot{\phi}_{2}, \mathcal{L} \dot{z}^{N}\right)+\left(R_{3}, 2 \mathcal{L} \dot{z}^{N}\right)+e_{1},
\end{aligned}
$$

where the $e_{1}$ term is bounded by

$$
\left|e_{1}\right| \leq\left\|\dot{\phi}_{1}\right\|_{H^{1}}^{2}+\left\|\dot{\phi}_{2}\right\|_{H^{1}}^{2}+\left\|\dot{z}^{N}\right\|_{H^{1}}^{2}+\|\dot{\Gamma}\|_{H^{1 / 2}}^{2} .
$$

REMARK 5.1. In deriving the left hand side of (77), we have used Lemma 5.1. Using $\mathcal{L}^{*}=\mathcal{L}+E_{0}$, we have

$$
\begin{aligned}
\left(\mathcal{L} \dot{z}^{N}, \dot{z}_{t}^{N}\right)+\left(\mathcal{L} \dot{z}^{N}, \dot{z}_{t}^{N}\right) & =\left(\mathcal{L} \dot{z}^{N}, \dot{z}_{t}^{N}\right)+\left(\dot{z}^{N}, \mathcal{L}^{*} \dot{z}_{t}^{N}\right) \\
& =\left(\mathcal{L} \dot{z}^{N}, \dot{z}_{t}^{N}\right)+\left(\dot{z}^{N}, \mathcal{L} \dot{z}_{t}^{N}\right)+\left(\dot{z}^{N}, E_{0}\left(\dot{z}_{t}^{N}\right)\right) \\
& =\frac{d}{d t}\left(\mathcal{L} \dot{z}^{N}, \dot{z}^{N}\right)-\left(\mathcal{L}_{t} \dot{z}^{N}, \dot{z}^{N}\right)+\left(\dot{z}^{N}, E_{0}\left(\dot{z}_{t}^{N}\right)\right) .
\end{aligned}
$$

Since the coefficients in $\mathcal{L}$ are smooth and independent of the perturbed quantities, it is easy to show by direct calculations that $\mathcal{L}_{t}$ is a singular integral operator of the same order as $\mathcal{L}$. To illustrate, we may consider the time derivative of $\Lambda f$ :

$$
\Lambda_{t} f=-\frac{3}{2} \int \frac{\left(\left(\left|z_{\alpha_{1}}(\alpha)\right|^{2}\right)_{t}\left(\alpha_{1}-\alpha_{1}^{\prime}\right)^{2}+\left(\left|z_{\alpha_{2}}(\alpha)\right|^{2}\right)_{t}\left(\alpha_{2}-\alpha_{2}^{\prime}\right)^{2}\right)\left(f(\alpha)-f\left(\alpha^{\prime}\right)\right)}{\left(\left|z_{\alpha_{1}}(\alpha)\right|^{2}\left(\alpha_{1}-\alpha_{1}^{\prime}\right)^{2}+\left|z_{\alpha_{2}}(\alpha)\right|^{2}\left(\alpha_{2}-\alpha_{2}^{\prime}\right)^{2}\right)^{5 / 2}} d \alpha^{\prime} .
$$


Clearly the resulting kernel of $\Lambda_{t}$ is of the same order as that of $\Lambda$. Similar observation applies to other operators. Thus $\left(\mathcal{L}_{t} \dot{z}^{N}, \dot{z}^{N}\right)$ and $\left(\dot{z}^{N}, E_{0}\left(\dot{z}_{t}^{N}\right)\right)$ can be bounded like the $e_{1}$ term mentioned above.

Next we multiply by $2\left(\sigma_{1} \sigma_{2}\right)^{2} \mathcal{L} \dot{\phi}_{i}$ to the $\dot{\phi}_{i}$ equation $(i=1,2)$. We obtain

$$
\begin{aligned}
\frac{d}{d t}\left(\dot{\phi}_{1},\left(\sigma_{1} \sigma_{2}\right)^{2} \mathcal{L} \dot{\phi}_{1}\right)= & \left.\left(2\left(\sigma_{1} \sigma_{2}\right)^{2} \mathcal{L} \dot{\phi}_{1}, R_{1}\right)\right)+e_{2}, \\
\frac{d}{d t}\left(\dot{\phi}_{2},\left(\sigma_{1} \sigma_{2}\right)^{2} \mathcal{L} \dot{\phi}_{2}\right)= & -\left(\left(\sigma_{1} \sigma_{2}\right)^{3} \widetilde{\Lambda}\left(\gamma_{1} H_{1}+\gamma_{2} H_{2}\right) \dot{z}^{N}, \mathcal{L} \dot{\phi}_{2}\right) \\
& +\left(2\left(\sigma_{1} \sigma_{2}\right)^{2} \mathcal{L} \dot{\phi}_{2}, R_{2}\right)+e_{3},
\end{aligned}
$$

where $e_{2}$ and $e_{3}$ can be bounded like the $e_{1}$ term. Hereafter, we will denote by $e_{i}$ the lower order terms that can be bounded in the same way as the $e_{1}$ term.

In the $\dot{\Gamma}$ equation, we multiply by $\widetilde{\Lambda} \dot{\Gamma}$ and integrate with respect to $\alpha$ to obtain

$$
\frac{1}{2} \frac{d}{d t}(\dot{\Gamma}, \widetilde{\Lambda} \dot{\Gamma})=\left(\widetilde{\Lambda} \dot{\Gamma}, \sigma_{1} \sigma_{2} \mathcal{L} \dot{z}^{N}\right)+\left(\widetilde{\Lambda} \dot{\Gamma}, R_{4}\right)+e_{4}
$$

where we have used the fact that $\widetilde{\Lambda}$ is self-adjoint (Lemma 5.2). Combining estimates from (77) to (80), and noting that leading order terms cancel each other, we have

$$
\begin{aligned}
& \frac{d}{d t}\left\{\left(\dot{\phi}_{1},\left(\sigma_{1} \sigma_{2}\right)^{2} \mathcal{L} \dot{\phi}_{1}\right)+\left(\dot{\phi}_{2},\left(\sigma_{1} \sigma_{2}\right)^{2} \mathcal{L} \dot{\phi}_{2}\right)+\left(\dot{z}^{N}, \mathcal{L} \dot{z}^{N}\right)+\frac{1}{2}\left(\dot{\Gamma},\left(\sigma_{1} \sigma_{2}\right)^{-1} \widetilde{\Lambda} \dot{\Gamma}\right)\right\} \\
& =\left(2\left(\sigma_{1} \sigma_{2}\right)^{2} \mathcal{L} \dot{\phi}_{1}, R_{1}\right)+\left(2\left(\sigma_{1} \sigma_{2}\right)^{2} \mathcal{L} \dot{\phi}_{2}, R_{2}\right)+\left(\mathcal{L} \dot{z}^{N}, R_{3}\right)+\left(\widetilde{\Lambda} \dot{\Gamma}, R_{4}\right)+e_{5} .
\end{aligned}
$$

\section{Step 3. Energy estimate for lower order terms.}

The lower order terms, $R_{i}(i=1, . ., 4)$, consist of three types of terms. The first type is in the form of $E_{0}(\dot{\phi})+E_{0}(\dot{\mathbf{z}})$. The second type is the convection term such as $\mathbf{w} \cdot \nabla \dot{\phi}_{i}$. The third type is given by $\tilde{K}_{i}(\mathrm{i}=1,2)$ in the $\dot{\phi}_{i}$ equations. The $\tilde{K}_{i}$ terms correspond to the new difficulty for 3 -D surface problems. In the 2-D case, the corresponding $\tilde{K}_{i}$ term is an infinitely order smoothing operator. But in $3-\mathrm{D}$, it is only a bounded operator. This term requires a special technique to control its growth. We will leave the estimate of the $\tilde{K}_{i}$ towards the end of the energy estimate (Step 5).

For the first type of terms, it is easy to show that

$$
\left|\left(2\left(\sigma_{1} \sigma_{2}\right)^{2} \mathcal{L} \dot{\phi}_{i}, E_{0}(\dot{\phi})+E_{0}(\dot{\mathbf{z}})\right)\right| \leq e_{1} .
$$

The convection terms can also be bounded like $e_{1}$. To see this, we consider the leading order term of the form $\left(w_{i} D_{i} \dot{z}^{N}, \sigma_{j} D_{j} \sigma_{j} D_{j} \dot{z}^{N}\right)$. Using integration by parts twice, we get

$$
\left(w_{i} D_{i} \dot{z}^{N}, \sigma_{j} D_{j} \sigma_{j} D_{j} \dot{z}^{N}\right)=\int w_{i} D_{i}\left(\left(\sigma_{j} D_{j} z^{N}\right)^{2}\right) d \alpha+e_{1}=e_{1}
$$

where we have used the fact that $\mathbf{w}$ is a smooth function depending on $\mathbf{z}$ only. We have also used $e_{1}$ symbolically to denote lower order terms that can be bounded like $e_{1}$.

The convection term in the $\dot{\Gamma}$ equation is in a slightly different form. Using Lemma 5.2 , Lemma 4.2 , and the fact that the commutator operator, $\left[\widetilde{\Lambda}, D_{i}\right]$, is an order one operator, we have

$$
\begin{aligned}
\left(\widetilde{\Lambda} \dot{\Gamma}, w_{i} D_{i} \dot{\Gamma}\right)= & \left(\dot{\Gamma}, \widetilde{\Lambda}\left(w_{i} D_{i}\right) \dot{\Gamma}\right)=\left(\dot{\Gamma}, w_{i} \widetilde{\Lambda} D_{i} \dot{\Gamma}\right)+e_{1} \\
& =\left(\dot{\Gamma}, w_{i} D_{i} \widetilde{\Lambda} \dot{\Gamma}\right)+e_{1}=-\left(w_{i} D_{i} \dot{\Gamma}, \widetilde{\Lambda} \dot{\Gamma}\right)+e_{1}
\end{aligned}
$$


This implies that

$$
\left(\widetilde{\Lambda} \dot{\Gamma}, w_{i} D_{i} \dot{\Gamma}\right)=\frac{1}{2} e_{1}
$$

All other convection terms can be treated similarly. We will not repeat this argument hereafter. Thus we have

$$
\begin{aligned}
& \frac{d}{d t}\left\{\left(\dot{\phi}_{1},\left(\sigma_{1} \sigma_{2}\right)^{2} \mathcal{L} \dot{\phi}_{1}\right)+\left(\dot{\phi}_{2},\left(\sigma_{1} \sigma_{2}\right)^{2} \mathcal{L} \dot{\phi}_{2}\right)+\left(\dot{z}^{N}, \mathcal{L} \dot{z}^{N}\right)+\frac{1}{2}\left(\dot{\Gamma},\left(\sigma_{1} \sigma_{2}\right)^{-1} \tilde{\Lambda} \dot{\Gamma}\right)\right\} \\
& =\left(2\left(\sigma_{1} \sigma_{2}\right)^{2} \mathcal{L} \dot{\phi}_{1}, \tilde{K}_{1}(\dot{\mu})\right)+\left(2\left(\sigma_{1} \sigma_{2}\right)^{2} \mathcal{L} \dot{\phi}_{2}, \tilde{K}_{2}(\dot{\mu})\right)+e_{6}
\end{aligned}
$$

\section{Step 4. Energy estimate in $L^{2}$ norm.}

We now try to obtain estimates for $\dot{\Gamma}$ in $L^{2}$ norm. Let

$$
\mathcal{L}_{0}=-\frac{\tau}{2}\left\{\left(\sigma_{2}\right)^{-1} D_{1}\left(\sigma_{1} D_{1}\right)+\left(\sigma_{1}\right)^{-1} D_{2}\left(\sigma_{2} D_{2}\right)-\Gamma_{1}^{12} D_{2}-\Gamma_{2}^{21} D_{1}\right\}
$$

The strategy for our energy estimate is as follows. To illustrate the idea, let us assume that $\tilde{\Lambda}$ is invertible. If we multiply the $\dot{z}^{N}$ equation by $\left(\sigma_{1} \sigma_{2}\right)^{-1} \mathcal{L}_{0} \tilde{\Lambda}^{-1}$, and multiply the $\dot{\Gamma}$ equation by $\dot{\Gamma}$, and add the resulting equations, then the leading order terms will cancel each other. Of course, $\tilde{\Lambda}$ is not invertible. But we can define an approximate inverse operator of $\widetilde{\Lambda}$ as follows:

$$
\widetilde{\Lambda}^{-1} f(\alpha) \equiv \sigma_{1} \sigma_{2} \int \frac{\left|\mathbf{z}_{\alpha_{1}}\right|\left|\mathbf{z}_{\alpha_{2}}\right|(\hat{f}(\xi)-\hat{f}(0))}{\left(\left|\mathbf{z}_{\alpha_{2}}\right|^{2} \xi_{1}^{2}+\left|\mathbf{z}_{\alpha_{1}}\right|^{2} \xi_{2}^{2}\right)^{1 / 2}} d \xi .
$$

It is easy to see that $\widetilde{\Lambda}^{-1}$ is well defined and is a smoothing operator of order one. Note that the commutator $\left[\widetilde{\Lambda}^{-1}, g\right]$ is a smoothing operator of order two for smooth $g$. Using the fact that $\widetilde{\Lambda}=\left(\sigma_{1} \sigma_{2}\right)^{-2} \Lambda+E_{0}$, we can show that $\widetilde{\Lambda}^{-1} \widetilde{\Lambda}(f)=f+E_{-1}(f)$.

Let $\left(\tilde{\Lambda}^{-1}\right)^{*}$ be the adjoint operator of $\tilde{\Lambda}^{-1}$. To obtain energy estimates, we multiply the $\dot{\Gamma}$ equation by $d \dot{\Gamma}$ and integrate with respect to $\alpha$. Next we multiply the $\dot{z}^{N}$ equation by $2 d\left(\sigma_{1} \sigma_{2}\right)^{-1}\left(\widetilde{\Lambda}^{-1}\right)^{*} \mathcal{L}_{0} \dot{z}^{N}$, and integrate with respect to $\alpha$. Similarly, for the $\dot{\phi}_{i}$ equations, we multiply by $2 d\left(\sigma_{1} \sigma_{2}\right)\left(\widetilde{\Lambda}^{-1}\right)^{*} \mathcal{L}_{0} \dot{\phi}_{i}$, and integrate with respect to $\alpha$. Using the property of $\tilde{\Lambda}^{-1}$ and Lemma 4.2 , we obtain

$$
\begin{aligned}
& \frac{d}{d t}\left\{\left(\dot{\phi}_{1}, d\left(\sigma_{1} \sigma_{2}\right)\left(\tilde{\Lambda}^{-1}\right)^{*} \mathcal{L}_{0} \dot{\phi}_{1}\right)+\left(\dot{\phi}_{2}, d\left(\sigma_{1} \sigma_{2}\right)\left(\tilde{\Lambda}^{-1}\right)^{*} \mathcal{L}_{0} \dot{\phi}_{2}\right)\right. \\
& \left.+\left(\dot{z}^{N}, d\left(\sigma_{1} \sigma_{2}\right)^{-1}\left(\tilde{\Lambda}^{-1}\right)^{*} \mathcal{L}_{0} \dot{z}^{N}\right)+\frac{d}{2}(\dot{\Gamma}, \dot{\Gamma})\right\}=e_{7}
\end{aligned}
$$

Here the constant $d>0$ is chosen so that $(\dot{\Gamma},(\widetilde{\Lambda}+d I) \dot{\Gamma})$ defines a generalized $H^{1 / 2}$ norm, see Eq. (4.32) in [16]. In obtaining the above estimate, we have used the fact that $\left(\widetilde{\Lambda}^{-1}\right)^{*}=\left(\widetilde{\Lambda}^{-1}\right)+E_{-2}, \mathcal{L}_{0}^{*}=\mathcal{L}_{0}+E_{0}$, and $\left(\widetilde{\Lambda}^{-1}\right)^{*} \mathcal{L}_{0}$ is self adjoint to the leading order, i.e.

$$
\left\{\left(\widetilde{\Lambda}^{-1}\right)^{*} \mathcal{L}_{0}\right\}^{*}=\mathcal{L}_{0}^{*}\left(\tilde{\Lambda}^{-1}\right)=\left(\widetilde{\Lambda}^{-1}\right) \mathcal{L}_{0}^{*}+E_{0}=\left(\widetilde{\Lambda}^{-1}\right)^{*} \mathcal{L}_{0}+E_{0} .
$$

Consequently, we have

$$
\begin{aligned}
2\left(\dot{z}_{t}^{N}, d\left(\sigma_{1} \sigma_{2}\right)^{-1}\left(\widetilde{\Lambda}^{-1}\right)^{*} \mathcal{L}_{0} \dot{z}^{N}\right) & =\left(\dot{z}_{t}^{N}, d\left(\sigma_{1} \sigma_{2}\right)^{-1}\left(\widetilde{\Lambda}^{-1}\right)^{*} \mathcal{L}_{0} \dot{z}^{N}\right) \\
& +\left(\dot{z}^{N}, d\left(\sigma_{1} \sigma_{2}\right)^{-1}\left(\widetilde{\Lambda}^{-1}\right)^{*} \mathcal{L}_{0} \dot{z}_{t}^{N}\right)+\left(\dot{z}^{N}, E_{0}\left(\dot{z}_{t}^{N}\right)\right) \\
& =\frac{d}{d t}\left(\dot{z}^{N}, d\left(\sigma_{1} \sigma_{2}\right)^{-1}\left(\widetilde{\Lambda}^{-1}\right)^{*} \mathcal{L}_{0} \dot{z}^{N}\right)+e_{8}
\end{aligned}
$$


by using the $\dot{z}_{t}^{N}$ equation. It is worth noting that $\left(\widetilde{\Lambda}^{-1}\right)^{*} \mathcal{L}_{0}$ is a linear operator of order one and is self-adjoint up to a bounded operator.

In order to accommodate the destabilizing term in the $\mathcal{L}$ operator, we need to obtain an estimate in the $L^{2}$-norm for $\dot{\mathbf{z}}$ in order to take care of the lower modes. Multiplying by $2(M+\tau) \dot{z}^{N}$ to the $\dot{z}^{N}$ equation and by $2(M+\tau) \dot{\phi}_{i}$ to the $\dot{\phi}_{i}$ equations, we obtain

$$
(M+\tau) \frac{d}{d t}\|\dot{\mathbf{z}}\|_{L^{2}}^{2}=e_{9}
$$

where $M$ is a positive constant to be determined later.

\section{Step 5. Estimate of $\widetilde{K}_{i}(\dot{\mu})$ terms.}

It remains to take care of the $\widetilde{K}_{i}(\dot{\mu})$ term in (81). It is important that $\widetilde{K}_{i}(\dot{\mu})$ terms only appear in the $\dot{\phi}_{i}$ equations, but not in the $\dot{z}^{N}$ equation. The reason we have introduced the $\tilde{\Lambda}$ operator in the $\dot{z}^{N}$ equation is to avoid having a term like $\widetilde{K}_{i}(\dot{\mu})$ in the $\dot{z}^{N}$ equation.

The main idea of controlling the $\widetilde{K}_{i}(\dot{\mu})$ term is to introduce some first order cross terms from the $\dot{z}^{N}$ equation to cancel the contribution of the $\widetilde{K}_{i}(\dot{\mu})$ terms in the $\dot{\phi}_{i}$ equations. And these first order cross terms can in turn be bounded by the $H^{1}$ norm and the $L^{2}$ norm in $\dot{\phi}$ and $\dot{z}^{N}$.

To be more specific, we first introduce

$$
\mathcal{N}_{i}=4\left(\sigma_{1} \sigma_{2}\right)\left(\widetilde{\Lambda}^{-1}\right)^{*} \widetilde{K}_{i}^{*} \mathcal{L}, \quad i=1,2 .
$$

Clearly, $\mathcal{N}_{i}$ is a first order operator. Multiplying the $\dot{z}^{N}$ equation by $\mathcal{N}_{i} \dot{\phi}_{i}$ and integrating with respect to $\alpha$, we obtain using the definition of $\dot{\Gamma}$

$$
\begin{aligned}
\frac{d}{d t}\left(\mathcal{N}_{i} \dot{\phi}_{i}, \dot{z}^{N}\right) & =-\left(\frac{1}{2}\left(\sigma_{1} \sigma_{2}\right) \tilde{\Lambda}(\dot{\mu}), 4 \sigma_{1} \sigma_{2}\left(\tilde{\Lambda}^{-1}\right)^{*} \tilde{K}_{i}^{*} \mathcal{L} \dot{\phi}_{i}\right)+e_{8} \\
& =-\left(2\left(\sigma_{1} \sigma_{2}\right)^{2} \tilde{K}_{i} \dot{\mu}, \mathcal{L} \dot{\phi}_{i}\right)+e_{8}
\end{aligned}
$$

where we have used the fact that

$$
\left|\frac{d}{d t}\left(\mathcal{N}_{i} \dot{\phi}_{i}, \dot{z}^{N}\right)-\left(\mathcal{N}_{i} \dot{\phi}_{i}, \dot{z}_{t}^{N}\right)\right|=\left|\left(\left(\mathcal{N}_{i}\left(\dot{\phi}_{i}\right)\right)_{t}, \dot{z}^{N}\right)\right| \leq C\left|e_{1}\right|
$$

using the $\dot{\phi}_{i}$ equation. The right hand sides of (87), when added to the right hand side of (83), will cancel the contribution from the $\widetilde{K}_{i}$ terms. The left hand side can be controlled by the $H^{1}$ norm of $\dot{\phi}_{i}$ and $\dot{z}^{N}$, as will be seen below.

\section{Step 6. Final energy estimates.}

Finally, we can combine the estimates (83), (85), (86), and (87) to obtain our desired energy estimates

$$
\frac{d}{d t} y_{1}(t) \leq C\left(\sum_{i=1}^{2}\left\|\dot{\phi}_{i}\right\|_{H^{1}}^{2}+\left\|\dot{z}^{N}\right\|_{H^{1}}^{2}+\|\dot{\Gamma}\|_{H^{1 / 2}}^{2}\right)
$$

where

$$
\begin{aligned}
y_{1}(t)= & \sum_{i=1}^{2}\left(\dot{\phi}_{i},\left(\sigma_{1} \sigma_{2}\right)^{2} \widetilde{\mathcal{L}} \dot{\phi}_{i}\right)+\left(\dot{z}^{N}, \widetilde{\mathcal{L}}^{N}\right)+\frac{1}{2}(\dot{\Gamma},(\widetilde{\Lambda}+d I) \dot{\Gamma})+(M+\tau)\|\dot{\mathbf{z}}\|_{L^{2}}^{2} \\
& +\sum_{i=1}^{2}\left(\mathcal{N}_{i} \dot{\phi}_{i}, \dot{z}^{N}\right)
\end{aligned}
$$


$\widetilde{\mathcal{L}}=\mathcal{L}+d\left(\tilde{\Lambda}^{-1}\right)^{*} \mathcal{L}_{0}$, and $\|\dot{\Gamma}\|_{H^{1 / 2}}^{2}=(\dot{\Gamma},(\widetilde{\Lambda}+d I) \dot{\Gamma})$. To close the energy estimates, we need to show that by appropriate choice of $M, y_{1}(t)$ is equivalent to the norm of the state variables, i.e.

$$
C_{1} y_{1}(t) \leq\left\|\dot{\phi}_{1}\right\|_{H^{1}}^{2}+\left\|\dot{\phi}_{2}\right\|_{H^{1}}^{2}+\left\|\dot{z}^{N}\right\|_{H^{1}}^{2}+\|\dot{\Gamma}\|_{H^{1 / 2}}^{2} \leq C_{2} y_{1}(t) .
$$

The first inequality is easy to obtain by using Lemma 4.5 and Lemma 4.2. To prove the second inequality, i.e. the lower bound, we note that

$$
\left(\dot{z}^{N}, \widetilde{\mathcal{L}} \dot{z}^{N}\right)=\frac{\tau}{2} \sum_{i=1}^{2}\left(\sigma_{i} D_{i} \dot{z}^{N},\left(\sigma_{1} \sigma_{2}\right)^{-1} \sigma_{i} D_{i} \dot{z}^{N}\right)+\left(\dot{z}^{N}, \mathcal{N}_{3} \dot{z}^{N}\right)
$$

where $\mathcal{N}_{3}$ is a first order operator. Using the Schwarz inequality and the fact that $2 a b \leq\left(\frac{a}{\epsilon_{0}}+\epsilon_{0} b\right)$, we obtain

$$
\begin{aligned}
\left|\left(\dot{z}^{N}, \mathcal{N}_{3} \dot{z}^{N}\right)\right| & \leq C\left\|\dot{z}^{N}\right\|_{L^{2}} \cdot\left\|\dot{z}^{N}\right\|_{H^{1}} \leq \frac{C}{2}\left(\frac{1}{\varepsilon_{0}}\left\|\dot{z}^{N}\right\|_{L^{2}}^{2}+\varepsilon_{0}\left\|\dot{z}^{N}\right\|_{H^{1}}^{2}\right), \\
\left|\left(\dot{\phi}_{i}, \mathcal{N}_{3} \dot{\phi}_{i}\right)\right| & \leq C\left\|\dot{\phi}_{i}\right\|_{L^{2}} \cdot\left\|\dot{\phi}_{i}\right\|_{H^{1}} \leq \frac{C}{2}\left(\frac{1}{\varepsilon_{0}}\left\|\dot{\phi}_{i}\right\|_{L^{2}}^{2}+\varepsilon_{0}\left\|\dot{\phi}_{i}\right\|_{H^{1}}^{2}\right), \\
\left|\left(\dot{z}^{N}, \mathcal{N}_{i} \dot{\phi}_{i}\right)\right| & \leq C\left\|\dot{z}^{N}\right\|_{L^{2}} \cdot\left\|\dot{\phi}_{i}\right\|_{H^{1}} \leq \frac{C}{2}\left(\frac{1}{\varepsilon_{0}}\left\|\dot{z}^{N}\right\|_{L^{2}}^{2}+\varepsilon_{0}\left\|\dot{\phi}_{i}\right\|_{H^{1}}^{2}\right), \quad i=1,2,
\end{aligned}
$$

where $\varepsilon_{0}$ is a small parameter to be determined later. Now the lower bound of $y_{1}$ can be estimated as follows

$$
\begin{aligned}
C_{2} y_{1} & \geq \tau\left\|\dot{z}^{N}\right\|_{H^{1}}^{2}+\tau\|\dot{\phi}\|_{H^{1}}^{2}+\frac{1}{2}\|\dot{\Gamma}\|_{H^{1 / 2}}^{2}+M\|\dot{\mathbf{z}}\|_{L^{2}}^{2} \\
& -\frac{2 C}{\epsilon_{0}}\left\|\dot{z}^{N}\right\|_{L^{2}}^{2}-\frac{C}{\epsilon_{0}}\|\dot{\phi}\|_{L^{2}}^{2}-C \epsilon_{0}\left\|\dot{z}^{N}\right\|_{H^{1}}^{2}-2 C \epsilon_{0}\|\dot{\phi}\|_{H^{1}}^{2} \\
& \geq\left(\tau-2 C \epsilon_{0}\right)\left\|\dot{z}^{N}\right\|_{H^{1}}^{2}+\left(\tau-2 C \epsilon_{0}\right)\|\dot{\phi}\|_{H^{1}}^{2} \\
& +\left(M-\frac{2 C}{\epsilon_{0}}\right)\|\dot{\mathbf{z}}\|_{L^{2}}^{2}+\frac{1}{2}\|\dot{\Gamma}\|_{H^{1 / 2}}^{2} .
\end{aligned}
$$

Now if we choose $\epsilon_{0}=\frac{\tau}{4 C}$ and $M=\frac{4 C^{2}}{\tau}+1$, then we obtain the desired estimate

$$
C_{2} y_{1} \geq \min \left(\frac{\tau}{2}, \frac{1}{2}\right)\left(\|\dot{\mathbf{z}}\|_{H^{1}}^{2}+\|\dot{\Gamma}\|_{H^{1 / 2}}^{2}\right) \text {. }
$$

This in turn implies that

$$
\frac{d}{d t} y_{1}(t) \leq \tilde{C} y_{1}(t)
$$

where $\tilde{C}$ depends on $\tau^{-1}$ and the regularity of the unperturbed smooth solution. The theorem now follows from the Gronwall inequality, i.e. there exists a $\widetilde{B}(t)$ so that

$$
\begin{aligned}
& \left\|\dot{\phi}_{1}(t)\right\|_{H^{1}}^{2}+\left\|\dot{\phi}_{2}(t)\right\|_{H^{1}}^{2}+\left\|\dot{z}^{N}(t)\right\|_{H^{1}}^{2}+\|\dot{\Gamma}(t)\|_{H^{1 / 2}}^{2} \\
& \leq \widetilde{B}(t)\left(\left\|\dot{\phi}_{1}(0)\right\|_{H^{1}}^{2}+\left\|\dot{\phi}_{2}(0)\right\|_{H^{1}}^{2}+\left\|\dot{z}^{N}(0)\right\|_{H^{1}}^{2}+\|\dot{\Gamma}(0)\|_{H^{1 / 2}}^{2}\right) .
\end{aligned}
$$

In terms of the original variables, we have

$$
\|\dot{\mathbf{z}}(t)\|_{H^{1}}^{2}+\|\dot{\mu}(t)\|_{H^{1 / 2}}^{2} \leq B(t)\left(\|\dot{\mathbf{z}}(0)\|_{H^{1}}^{2}+\|\dot{\mu}(0)\|_{H^{1 / 2}}^{2}\right) .
$$


This completes the proof of Theorem 3.1 in the case of $A=0$. The case of $A \neq 0$ will be considered next.

\section{Case II. Energy estimate for $A \neq 0$.}

The main idea of analysis for the case $A=0$ can be used to analyze the general case. As in the case of $A=0$, we introduce the new tangential variables $\dot{\phi}_{1}$ and $\dot{\phi}_{2}$ to eliminate one of the tangential variables up to leading order. We also introduce $\dot{\mu}$ as the new variable instead of using $\dot{\gamma}_{1}$ and $\dot{\gamma}_{2}$. Note that only the $\dot{\gamma}_{i}$ equation (38) is related to the Atwood number, $A$. So the leading order perturbed equations for $\dot{\phi}_{1}$, $\dot{\phi}_{2}$ and $\dot{z}^{N}$ are the same as in the case of $A=0$. We have

$$
\begin{aligned}
\frac{D \dot{\phi}_{1}}{D t}= & E_{0}(\dot{\phi})+E_{0}\left(\dot{z}^{N}\right)+\widetilde{K}_{1}(\dot{\mu}) \\
\frac{D \dot{\phi}_{2}}{D t}= & -\frac{1}{2} \sigma_{1} \sigma_{2} \widetilde{\Lambda}\left(\gamma_{1} H_{1}+\gamma_{2} H_{2}\right) \dot{z}^{N}+E_{0}(\dot{\phi})+E_{0}\left(\dot{z}^{N}\right)+\widetilde{K}_{2}(\dot{\mu}) \\
\frac{D \dot{z}^{N}}{D t}= & \frac{1}{2} \sigma_{1} \sigma_{2} \widetilde{\Lambda}(\dot{\mu})+\frac{1}{2}\left(\sigma_{1} \sigma_{2}\right)^{2} \widetilde{\Lambda}\left[\left(\sigma_{1}\right)^{2} \gamma_{1} H_{2}-\left(\sigma_{2}\right)^{2} \gamma_{2} H_{1}\right] \dot{\phi}_{1} \\
& +\frac{1}{2}\left(\sigma_{1} \sigma_{2}\right)^{3} \widetilde{\Lambda}\left(\gamma_{1} H_{1}+\gamma_{2} H_{2}\right) \dot{\phi}_{2}+E_{0}(\dot{\phi})+E_{0}\left(\dot{z}^{N}\right) \\
\frac{D \dot{\mu}}{D t}= & \mathcal{E}_{1}\left(\dot{\phi}_{1}\right)+\mathcal{E}_{2}\left(\dot{\phi}_{2}\right)-2 A K\left(\frac{\partial \dot{\mu}}{\partial t}\right)+\mathcal{M}(\dot{\mu}) \\
& +\frac{\tau}{2}\left\{\sigma_{1} D_{1}\left(\sigma_{1} D_{1} \dot{z}^{N}\right)+\sigma_{2} D_{2}\left(\sigma_{2} D_{2} \dot{z}^{N}\right)-\sigma_{1} \sigma_{2}\left(\Gamma_{1}^{12} \dot{z}_{\alpha_{2}}^{N}+\Gamma_{2}^{21} \dot{z}_{\alpha_{1}}^{N}\right)\right\} \\
& +E_{0}(\dot{\mathbf{z}})+E_{0}(\dot{\mu})
\end{aligned}
$$

where operators $\mathcal{E}_{i}, i=1,2$, and $\mathcal{M}$ are defined as

$$
\begin{aligned}
\mathcal{E}_{1}= & A\left\{-\left(\sigma_{1} \sigma_{2}\right)^{2} \sigma_{1}\left(\sigma_{2}\right)^{-1}\left[\gamma_{1}^{2} \sigma_{1}^{2} D_{1}+\gamma_{1} \gamma_{2} \sigma_{2}^{2} D_{2}\right] H_{2}\right. \\
& \left.+\left(\sigma_{1} \sigma_{2}\right)^{2}\left[\gamma_{1} \gamma_{2} \sigma_{1}^{2} D_{1}+\gamma_{2}^{2} \sigma_{2}^{2} D_{2}\right] H_{1}\right\} \\
\mathcal{E}_{2}= & A\left\{\left(\sigma_{1} \sigma_{2}\right)^{2}\left(\sigma_{1}\right)^{-1} \sigma_{2}\left[\gamma_{1}^{2} \sigma_{1}^{2} D_{1}+\gamma_{1} \gamma_{2} \sigma_{2}^{2} D_{2}\right] H_{1}\right. \\
& \left.-\left(\sigma_{1} \sigma_{2}\right)^{2}\left[\gamma_{1} \gamma_{2} \sigma_{1}^{2} D_{1}+\gamma_{2}^{2} \sigma_{2}^{2} D_{2}\right] H_{2}\right\} \\
\mathcal{M}= & \frac{A}{2}\left[\left(\sigma_{1} \sigma_{2}\right)^{-2}\left(\gamma_{1} H_{1}^{2}+\gamma_{2} H_{1} H_{2}\right)-\gamma_{1} \sigma_{1}^{2}\right] D_{1} \\
& \left.+\frac{A}{2}\left[\left(\sigma_{1} \sigma_{2}\right)^{-2}\left(\gamma_{2} H_{2}^{2}+\gamma_{1} H_{1} H_{2}\right)-\gamma_{2} \sigma_{2}^{2}\right] D_{2}\right\} .
\end{aligned}
$$

In order to see clearly how the surface tension effect stabilizes the Kelvin-Helmholtz instability, we introduce new variable $\dot{\Gamma}(64)$ to incorporate other tangential terms into our $\dot{\mu}$ variable. Using the same definition of $\mathcal{L}$ operator and lower order terms $R_{i}, i=1,2,3,4$ as before, the evolution equations become

$$
\begin{aligned}
\frac{\partial \dot{\phi}_{1}}{\partial t} & =R_{1} \\
\frac{\partial \dot{\phi}_{2}}{\partial t} & =-\frac{1}{2} \sigma_{1} \sigma_{2} \tilde{\Lambda}\left(\gamma_{1} H_{1}+\gamma_{2} H_{2}\right) \dot{z}^{N}+R_{2} \\
\frac{\partial \dot{z}^{N}}{\partial t} & =-\frac{1}{2} \sigma_{1} \sigma_{2} \tilde{\Lambda} \dot{\Gamma}-\frac{1}{2}\left(\sigma_{1} \sigma_{2}\right)^{3} \widetilde{\Lambda}\left(\gamma_{1} H_{1}+\gamma_{2} H_{2}\right) \dot{\phi}_{2}+R_{3} \\
\frac{\partial \dot{\Gamma}}{\partial t} & =\sigma_{1} \sigma_{2} \mathcal{L} \dot{z}^{N}+\widetilde{\mathcal{E}}_{1}\left(\dot{\phi}_{1}\right)+\widetilde{\mathcal{E}}_{2}\left(\dot{\phi}_{2}\right)+\mathcal{M}(\dot{\Gamma})-2 A K\left(\frac{\partial \dot{\Gamma}}{\partial t}\right)+R_{4}
\end{aligned}
$$


where $\widetilde{\mathcal{E}}_{i}, i=1,2$, are defined as

$$
\begin{aligned}
& \widetilde{\mathcal{E}}_{1}=\mathcal{E}_{1}+\left(\sigma_{1} \sigma_{2}\right)\left[\left(\sigma_{2}\right)^{2} \gamma_{2} H_{1}-\left(\sigma_{1}\right)^{2} \gamma_{1} H_{2}\right] \mathcal{M} \\
& \widetilde{\mathcal{E}}_{2}=\mathcal{E}_{2}-2\left(\sigma_{1} \sigma_{2}\right)^{2}\left(\gamma_{1} H_{1}+\gamma_{2} H_{2}\right) \mathcal{M} .
\end{aligned}
$$

Note that $\widetilde{\mathcal{E}}_{i}$ is still a first order operator like $\mathcal{E}_{i}$.

The double-layer potential operator $K$ is a smoothing operator of order one, $K f=E_{-1}(f)$, by the Lemma 4.3. Moreover, $K$ is a self adjoint operator up to a smoothing operator of order two.

LemMA 5.3. $K$ is self adjoint up to a smoothing operator of order two. That is, for any function $f$ in $H^{s}$, we have

$$
K^{*} f=K f+E_{-2}(f)
$$

Proof. By the definition of the double layer operator, we have

$$
\begin{aligned}
K f & =\int f\left(\alpha^{\prime}\right)\left(z_{\alpha_{1}} \times z_{\alpha_{2}}\right)\left(\alpha^{\prime}\right) \cdot \nabla_{z^{\prime}} G\left(z(\alpha)-z\left(\alpha^{\prime}\right)\right) d \alpha^{\prime} \\
& =\int f\left(\alpha^{\prime}\right)\left(z_{\alpha_{1}} \times z_{\alpha_{2}}\right)\left(\alpha^{\prime}\right) \cdot \frac{\sum_{i, j=1}^{2} z_{\alpha_{i} \alpha_{j}}\left(\alpha^{\prime}\right)\left(\alpha_{i}-\alpha_{i}^{\prime}\right)\left(\alpha_{j}-\alpha_{j}^{\prime}\right)}{\left|z(\alpha)-z\left(\alpha^{\prime}\right)\right|^{3}}+E_{-2}(f),
\end{aligned}
$$

from which it is easy to show that

$$
K^{*} f-K f=E_{-2}(f) .
$$

The energy estimate for the leading order terms is the same as the case $A=0$. We can first derive energy estimates for the leading order terms by using the $H^{1}$ norm for the $\dot{\phi}_{i}(i=1,2)$ and $\dot{z}^{N}$, and using the $H^{1 / 2}$ norm for $\dot{\Gamma}$. The energy estimate for lower order terms can be treated as the case $A=0$. The $\mathcal{M}(\dot{\Gamma})$ term is a generalized convection term. Using an argument similar to what we did for the convection term, $(\widetilde{\Lambda} \dot{\Gamma}, \mathbf{w} \cdot \nabla \dot{\Gamma})$, in Step 3 , we can show that $(\widetilde{\Lambda} \dot{\Gamma}, \mathcal{M}(\dot{\Gamma}))$ can be bounded by $e_{1}$. Let $y_{1}(t)$ be defined as in (88). Using the same argument as in the case of $A=0$, we have

$$
\frac{d y_{1}(t)}{d t}=\left(\widetilde{\mathcal{E}}_{1}\left(\dot{\phi}_{1}\right), \widetilde{\Lambda} \dot{\Gamma}\right)+\left(\widetilde{\mathcal{E}}_{2}\left(\dot{\phi}_{2}\right), \widetilde{\Lambda} \dot{\Gamma}\right)-\left(2 A K\left(\frac{\partial \dot{\Gamma}}{\partial t}\right), \widetilde{\Lambda} \dot{\Gamma}\right)+e_{9}
$$

The treatment of the $\widetilde{\mathcal{E}}_{i}\left(\dot{\phi}_{i}\right)$ terms is similar to that of the $\widetilde{K}_{i}(\dot{\mu})$ terms in Step 5 in the case of $A=0$. Multiplying the $\dot{z}^{N}$ equation (96) by $2\left(\sigma_{1} \sigma_{2}\right)^{-1} \widetilde{\mathcal{E}}_{i} \dot{\phi}_{i}$ and integrating with respect to $\alpha$, we obtain

$$
\begin{aligned}
\frac{d}{d t}\left(2\left(\sigma_{1} \sigma_{2}\right)^{-1} \widetilde{\mathcal{E}}_{i} \dot{\phi}_{i}, \dot{z}^{N}\right) & =-\left(\frac{1}{2}\left(\sigma_{1} \sigma_{2}\right) \widetilde{\Lambda}(\dot{\Gamma}), 2\left(\sigma_{1} \sigma_{2}\right)^{-1} \widetilde{\mathcal{E}}_{i} \dot{\phi}_{i}\right)+e_{9} \\
& =-\left(\tilde{\Lambda}(\dot{\Gamma}), \widetilde{\mathcal{E}}_{i} \dot{\phi}_{i}\right)+e_{9}
\end{aligned}
$$

where we have used the fact that

$$
\left|\frac{d}{d t}\left(2\left(\sigma_{1} \sigma_{2}\right)^{-1} \widetilde{\mathcal{E}}_{i} \dot{\phi}_{i}, \dot{z}^{N}\right)-\left(2\left(\sigma_{1} \sigma_{2}\right)^{-1} \widetilde{\mathcal{E}}_{i} \dot{\phi}_{i}, \dot{z}_{t}^{N}\right)\right| \leq e_{1},
$$

using the $\dot{\phi}_{i}$ equation. The right hand sides of (101), when added to the right hand side of (100), will cancel the contribution from the $\widetilde{\mathcal{E}}_{i}$ terms. 
We are left to consider the term $\left(2 A K\left(\frac{\partial \dot{\Gamma}}{\partial t}\right), \widetilde{\Lambda} \dot{\Gamma}\right)$. This term corresponds to another new difficulty for 3 -D surface problems. It has been shown that $\frac{1}{2} I+K$ is invertible $[19,20]$. Thus $\frac{1}{2} I+A K$ is also invertible since the Atwood number $|A| \leq 1$. It follows from (97) that

$$
\frac{\partial \dot{\Gamma}}{\partial t}=E_{2}(\dot{\mathbf{z}})+E_{1}(\dot{\Gamma})
$$

Next we observe that

$$
\begin{aligned}
\frac{d}{d t}(A K \dot{\Gamma}, \widetilde{\Lambda} \dot{\Gamma}) & =\left(A K \dot{\Gamma}_{t}, \widetilde{\Lambda} \dot{\Gamma}\right)+\left(A K \dot{\Gamma}, \widetilde{\Lambda} \dot{\Gamma}_{t}\right)+e_{10} \\
& =\left(2 A K \dot{\Gamma}_{t}, \widetilde{\Lambda} \dot{\Gamma}\right)+\left(E_{-2}\left(\frac{\partial \dot{\Gamma}}{\partial t}\right), \widetilde{\Lambda} \dot{\Gamma}\right)+e_{10} \\
& =\left(2 A K \dot{\Gamma}_{t}, \widetilde{\Lambda} \dot{\Gamma}\right)+e_{10}
\end{aligned}
$$

where we have used (102) and the fact that $K$ is self adjoint upto an $E_{-2}$ operator. The right hand sides of (103), when added to the right hand side of (100), will cancel the contribution from the $-2 A K\left(\frac{\partial \dot{\Gamma}}{\partial t}\right)$ terms. The left hand side can be controlled by the $H^{1 / 2}$ norm of $\dot{\Gamma}$.

We now combine the estimates (100), (101) and (103) to obtain our desired energy estimates

$$
\frac{d}{d t} y_{2}(t) \leq C\left(\sum_{i=1}^{2}\left\|\dot{\phi}_{i}\right\|_{H^{1}}^{2}+\left\|\dot{z}^{N}\right\|_{H^{1}}^{2}+\|\dot{\Gamma}\|_{H^{1 / 2}}^{2}\right)
$$

where

$$
\begin{aligned}
y_{2}(t)= & \sum_{i=1}^{2}\left(\dot{\phi}_{i},\left(\sigma_{1} \sigma_{2}\right)^{2} \widetilde{\mathcal{L}} \dot{\phi}_{i}\right)+\left(\dot{z}^{N}, \widetilde{\mathcal{L}}^{N}\right)+\frac{1}{2}(\dot{\Gamma},(\widetilde{\Lambda}+d I) \dot{\Gamma})+(M+\tau)\|\dot{\mathbf{z}}\|_{L^{2}}^{2} \\
& +\sum_{i=1}^{2}\left(\mathcal{N}_{i} \dot{\phi}_{i}, \dot{z}^{N}\right)+\sum_{i=1}^{2}\left(2\left(\sigma_{1} \sigma_{2}\right) \widetilde{\mathcal{E}}_{i} \dot{\phi}_{i}, \dot{z}^{N}\right)+(A K \dot{\Gamma}, \widetilde{\Lambda} \dot{\Gamma})
\end{aligned}
$$

The last two terms in $y_{2}$ are new terms due to the contribution of $A \neq 0$ in the $\dot{\Gamma}$ equation. Recall that $K f=E_{-1}(f)$. Thus we have

$$
|(K \dot{\Gamma}, \tilde{\Lambda} \dot{\Gamma})| \leq C\|\dot{\Gamma}\|_{L^{2}}
$$

We choose $d$ sufficiently large so that

$$
(\dot{\Gamma}+2 A K \dot{\Gamma}, \tilde{\Lambda} \dot{\Gamma})+(\dot{\Gamma}, d \dot{\Gamma})
$$

defines a generalized $H^{1 / 2}$ norm. Note that $\widetilde{\mathcal{E}}_{i}$ is a first order operator. It can be combined to the $\mathcal{N}_{i}$ operator. The estimate of $\widetilde{\mathcal{E}}_{i}$ and $\mathcal{N}_{i}$ is the same as the case of $A=0$ using the Schwarz inequality. The other terms can be treated similar to the $A=0$ case. Therefore, $y_{2}(t)$ is equivalent to the norm of the state variables, i.e.

$$
\widetilde{C}_{1} y_{2}(t) \leq\left\|\dot{\phi}_{1}\right\|_{H^{1}}^{2}+\left\|\dot{\phi}_{2}\right\|_{H^{1}}^{2}+\left\|\dot{z}^{N}\right\|_{H^{1}}^{2}+\|\dot{\Gamma}\|_{H^{1 / 2}}^{2} \leq \widetilde{C}_{2} y_{2}(t)
$$

This implies that

$$
\frac{d}{d t} y_{2}(t) \leq \widetilde{C} y_{2}(t)
$$


The theorem now follows from the Gronwall inequality. In terms of the original variables, we have

$$
\|\dot{\mathbf{z}}(t)\|_{H^{1}}^{2}+\|\dot{\mu}(t)\|_{H^{1 / 2}}^{2} \leq B_{1}(t)\left(\|\dot{\mathbf{z}}(0)\|_{H^{1}}^{2}+\|\dot{\mu}(0)\|_{H^{1 / 2}}^{2}\right) .
$$

This completes the proof of Theorem 3.1.

Acknowledgements. It is a pleasure to thank Prof. Long-an Ying for many interesting and fruitful discussions. Hou's research is supported in part by ONR under the grant N00014-96-1-0438, by DOE under the grant DE-FG03-89ER25073, and by NSF under the grant DMS-9704976. Zhang's research is supported in part by a grant from the National Natural Science Foundation of China, and Doctoral Key grant from Educational Committee of China. Finally we would like to thank the reviewer for a careful reading of our original manuscript and many constructive comments which significantly improve the quality of this paper.

\section{REFERENCES}

[1] G. BAKER, Generalized vortex methods for free-surface flows, in Waves on Fluid Interfaces, R.E. Meyer ed., Academic Press, 53-81 (1983).

[2] G. BAKer, D. Meiron, AND S. ORSzAG, Generalized vortex methods for free-surface flow problems, J. Fluid Mech., 123, 477-501 (1982).

[3] J.T. Beale, T.Y. Hou And J.S. Lowengrub, Growth rates for the linear motion of fluid interfaces far from equilibrium, Comm. Pure Appl. Math., 46, 1269-1301 (1993).

[4] J.T. Beale, T.Y. Hou AND J.S. Lowengrub, Convergence of a boundary integral method for water waves, SIAM J. Num. Anal., 33, 1797-1843 (1996).

[5] J.T. Beale, T.Y. Hou AND J.S. Lowengrub, On the well-posedness of two fluid interfacial flows with surface tension, Proceeding of NATO Advanced Research Workshop on Singularities in Fluid, Plasma and Optics, Greece, R. Caflisch ed. (1992)

[6] J.T. Beale, T.Y. Hou And J.S. Lowengrub, Growth rates for the linearized motion of 2-D two-density fluid interfaces with surface tension far from equilibrium, unpublished note.

[7] R.C. Brower, D.A. Kessler, J. Koplik AND H. Levine, Geometrical models of interface evolution, Phys. Rev. A 29, (1984)

[8] R. CAFlisch, Mathematical analysis of vortex dynamics, in Mathematical Aspects of Vortex dynamics, Edited by R. Calflisch, SIAM Publications, (1989).

[9] R. CAFlisch, X. F. LI, AND M. Shelley, The Collapse of an axisymmetrical, swirling vortex sheet, Nonlinearity, 6, 843-867, (1993).

[10] H. Ceniceros AND T. Y. Hou, Convergence of a non-stiff boundary integral method for interfacial flows with surface tension, to appear in Math. Comp., in press.

[11] P. Drazin and W. Reid, Hydrodynamic Stability, Cambridge University Press, (1981).

[12] D. Haroldson and D. Meiron, Numerical Calculation of three Dimensional Interfacial Potential Flows using the Point Vortex Method, preprint, submitted to SIAM J. Sci. Comp. (1996).

[13] T. Y. Hou, Numerical solutions to free boundary problems, Acta Numerica, 335-415 (1995).

[14] T. Y. Hou, J. Lowengrub, AND M. Shelley, Removing the stiffness from interfacial flows with surface tension, J. Comput. Phys, 114, 312-338 (1994).

[15] T. Y. Hou, J. Lowengrub, And M. Shelley, The Long-Time Motion of Vortex Sheets with Surface Tension, Phys. of Fluid, A, 9, 1933-1954 (1997).

[16] T. Y. Hou, Z. H. Teng AND P. Zhang, Well-posedness of linearized motion for 3-D water waves far from equilibrium, Comm. in PDE, 21(9\&10), 1551-1585 (1996).

[17] T. Y. Hou AND P. ZHANG, Stability of boundary integral methods for 3-D water waves, in preparation

[18] Y. KANEDA, A representation of the motion of a vortex sheet in a three dimensional flow, Phys. Fluids A, 2, 458-461 (1990).

[19] C. E. Kenig, Recent progress on boundary value problems on Lipschitz domains, Proc. Symp. Pure Math., 43, 175-205 (1985).

[20] C. E. Kenig, Elliptic boundary value problems on Lipschitz domains, Ann. Math. Studies, $112,131-183$ (1986). 
[21] M. S. Longuet-Higgens AND E. D. Cokelet, The deformation of steep surface waves on water, I. A numerical method of computation, Proc. Roy. Soc. London A, 350, 1-26, (1976).

[22] M. NitSCHE AND R. KRASNY, A numerical study of vortex ring formation at the edge of a circular tube, J. Fluid Mech., 276, 139-161, (1994).

[23] J. Stoker, Differential Geometry, Pure and Applied Mathematics Vol XX Wiley-Interscience (1969).

[24] G. Strang, Necessary and sufficient conditions for well-posed Cauchy problems, J. Diff. Eq., V.2 (1966).

[25] G. I. TAYLOR, The instability of liquid surfaces when accelerated in a direction perpendicular ro their planes I, Proc. Roy. Soc. London A, 201, 192-196 (1950).

[26] H. Yosihara, Gravity waves on the free surface of an incompressible perfect fluid of finite depth, RIMS Kyoto 18, 49-96, (1982).

[27] P. ZhANG, On the Well-Posedness of 3-D Water Wave With Surface Tension, to appear, 1996. 